LETTER

\section{Mexiletine is effective on segmental hyperhidrosis: report of two cases}

Segmental hyperhidrosis occurs in patients who have spinal cord lesions such as syringomyelia, ${ }^{1}$ vascular disorders, or tumours. Severe segmental hyperhidrosis not only causes discomfort, but may disturb daily life, as it did with our patients. There have been no reports on the treatment of segmental hyperhidrosis. We report on two patients with segmental hyperhidrosis caused by syringomyelia and cavernous haemangioma of the spinal cord, in which oral administration of mexiletine ameliorated the symptoms.

A 41 year old woman experienced dysaesthesia of the left side of the trunk, which gradually progressed. At the age of 56 she developed dysaesthesia of the left upper limb as well. At that time, she had excessive sweating on the left side of her face, trunk, and upper limb, even when it was not hot, and had to change her nightwear a few times each night. Neurological examination detected dissociated sensory disturbance in the left half of the trunk in the Th2-Th4 area. Hyperhidrosis was present on the left side from the face to the trunk at the Th7 level. Deep tendon reflexes were normal with no pathological reflexes. This hyperhidrosis distribution was confirmed by the Mino test and quantified with a Servomed evaporimeter. Thermography showed reduced body temperature of the same distribution as hyperhidrosis. Radiography detected scoliosis at Thl-Th9. Magnetic resonance imaging showed syringomyelia prominent at Th2-Th4. No Chiari malformation was present. Her hyperhidrosis was relieved by the oral administration of $200 \mathrm{mg}$ /day mexiletine (fig 1). Oral administration of $400 \mathrm{mg} /$ day carbamazepine also gave partial relief. She did not have to change her nightwear and could sleep well.

A 56 year old man at first experienced increased sweating on both sides of the upper limbs and trunk, and from the age of 58 hyperhidrosis on his face. He had to change his clothes several times a day. His clinical symptoms slowly progressed, and at the age of

If you have a burning desire to respond to a paper published in JNNP why not make use of our "rapid response" option?

Log on to our website (www.jnnp. com), find the paper that interests you, and send your response via email by clicking on the "eletters" option in the box at the top right hand corner. Providing it isn't libellous or obscene, it will be posted within 7 days. You can retrieve it by clicking on "read eletters" on our homepage.

The editors will decide as before whether to also publish it in a future paper issue.

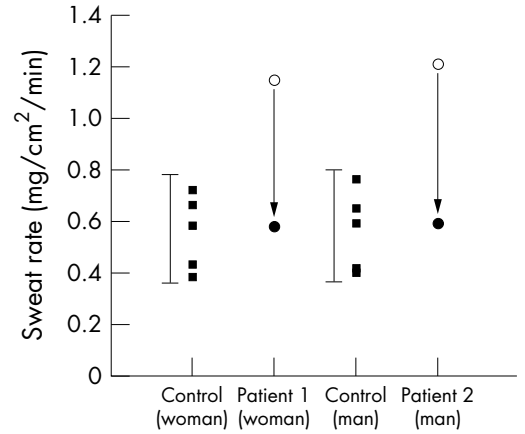

Figure 1 The sweat rate $\left(\mathrm{mg} / \mathrm{cm}^{2} / \mathrm{min}\right)$ was determined with a Kens-Perspiro OSS-100 (Suzuken Co Ltd.). All measurements were made at the Th4 level in the spine. The room was maintained at $60 \pm 1 \%$ humidity and $36 \pm 1^{\circ} \mathrm{C}$. Sweat rates were measured for both patients and five healthy men (mean age 53.6 years) and five women (mean age 42.3 years). Data for the patients were obtained before treatment (open circles ) and after oral administration of $200 \mathrm{mg} /$ day mexiletine (closed circles).

63, he was admitted to our hospital. Neurological examination showed bilateral hypaesthesia at Th2-Th5 and hyperhidrosis from the face to the dermatome Th10 level on both sides. Deep tendon reflexes were normal with no pathological reflexes. Hyperhidrosis was confirmed by the Minor test and quantified with a Servomed evaporimeter. Thermography showed decreased body temperature over the same area as the hyperhidrosis was distributed. Magnetic resonance imaging showed a cavernous haemangioma at Th2Th5. His hyperhidrosis was relieved by oral administration of $200 \mathrm{mg} /$ day mexiletine (fig 1). Oral administration of $400 \mathrm{mg} /$ day carbamazepine also gave partial relief. He did not have to change his clothes during the day.

In addition, we tried mexiletine to eight patients (five women, age range 18-34 years) with primary hyperhidrosis, which is defined as excessive, uncontrollable sweating without any discernible cause. No improvement was noted after oral administration of $200 \mathrm{mg} / \mathrm{day}$ mexiletine.

This is the first report of the use of mexiletine to treat segmental hyperhidrosis. Three mechanisms have been hypothesised as possible causes of segmental hyperhidrosis brought about by spinal cord lesions: (1) disinhibition of preganglionic sympathetic neurons due to interruption of the inhibitory descending pathway, ${ }^{2}$ (2) overactivity of preganglionic sympathetic neurons due to spinal cord lesions, ${ }^{3}$ and (3) autonomic hyperreflexia due to cutaneous stimulation, such as postural change, or bladder and intestine stimulation.

We judge that the mechanism of segmental hyperhidrosis in our patients was produced by overactivity of the preganglionic sympathetic neurons. The intermediolateral nucleus, located in the lateral horn of the spinal cord from C8-Th1 to L2-L4, receives both excitatory and inhibitory descending innervations from the thalamus. The face and neck are innervated by the intermediolateral nucleus at the Th2-Th4 level, in the upper limbs at Th2-Th8, in the trunk at Th6-Th10, and in the lower limbs at Th1 1-Thl2. The ambiguous, overlapping innervation by the intermediolateral nucleus accounts for the differences in the distributions of the sensory disturbances and hyperhidrosis caused by the same spinal cord lesion. In fact, the hyperhidrosis distributions in our patients corresponded to the area innervated by the intermediolateral nucleus at the levels of the lesions seen on MRI and of the sensory deficits. This hyperhidrosis distribution cannot be explained by interruption of the inhibitory descending pathway. Because hyperhidrosis in our patients occurred even without cutaneous stimulation, we suspect persistent spontaneous overactivity of the preganglionic sympathetic neurons due to spinal cord lesions.

Oral administration of mexiletine was an excellent remedy for the segmental hyperhidrosis of our patients. Mexiletine is a sodium channel blocker reported to be effective for treating painful neuropathies. ${ }^{5}$ In terms of its mechanism, mexiletine is thought to inhibit the spontaneous activity of regenerating fibres in the spinal cord or to act on the spinal mediated nociceptive flexor reflex. ${ }^{5}$ We consider it compatible that in our patients mexiletine inhibited the spontaneous activity of the intermediolateral nucleus caused by spinal cord leisons. Our findings indicate that sodium channel blockers, such as mexiletine and carbamazepine, should be considered for the treatment of segmental hyperhidrosis caused by spinal cord lesions.

S Ishibashi, T Yokota, A Inaba, M Yamada, H Mizusawa Department of Neurology, Tokyo Medical and Dental University School of Medicine, 1-5-45, Yushima, Bunkyo-ku, Tokyo 113-8519, Japan

T Iwai

Department of Surgery, Division of Vascular Surgery

T Shiojiri

Department of Neurology, Asahi General Hospital, I- 1326, Asahi, Chiba, 289-2511, Japan

Correspondence to: Dr T Yokota: tak-yokota.neuro@tmd.ac.jp

\section{References}

1 Sudo K, Naoto F, Tashiro K, et al. Focal (segmental) dyshidrosis in syringomyelia. J Neurol Neurosurg Psychiatry 1999;67:106-8.

2 Stovner LJ, Sjaastad O. Segmental hyperhidrosis in two siblings with Chiari type malformation. Eur Neurol 1995:35:149-55.

3 Sudo K, Tashiro K. Segmental hyperhidrosis in syringomyelia with Chiari malformation. J Neurol 1993;240:75-8.

4 Glasaver FE, Czyrny JJ. Hyperhidrosis as the presenting symptom in post traumatic syringomyelia. Paraplegia 1994;32:423-9.

5 Dejgard A, Petersen P, Kastrup J. Mexiletine for treatment of chronic painful diabetic neuropathy. Lancet 1988;i:9-11

Does the presence of a pontine trigeminal lesion represent an absolute contraindication for microvascular decompression in drug resistant trigeminal neuralgia?

Typical trigeminal neuralgia is characterised by recurring, paroxysmal, lancinating, shocklike pain within the distribution of one or 
more branches of the trigeminal nerve. Light tactile stimulation may trigger such an attack. Although the contribution of central and peripheral mechanisms to the aetiopathogenesis of trigeminal neuralgia still remains unclear, the concept of vascular compression of the trigeminal root as the main causal factor in idiophatic "tic douloureux" has achieved widespread acceptance. Trigeminal neuralgia may also afflict patients with multiple sclerosis. In these cases, the demyelination of central trigeminal pathways is the accepted aetiology, and the presence of a T2 hyperintensity along the intrapontine course of trigeminal fibres is generally considered a contraindication to microvascular decompression. We recently saw a case of successful microvascular decompression in a patien without multiple sclerosis, despite an intrapontine trigeminal lesion.

This 66 year old previously healthy man presented with a 6 year history of intense, paroxysmal, electric shock-like pain in the territory of the second branch of the right trigeminal nerve. The pain was triggered by washing his face and shaving and it lasted for a few seconds. Painful attacks, initially rare, gradually increased in frequency and intensity and spread to the first trigeminal branch. When admitted to our hospital $1200 \mathrm{mg}$ carbamazepine were ineffective in relieving the pain. Before admission phenitoin, baclofen, and lamotrigine had been tried without success. Neurological examination was negative and in particular there were no gross sensory deficits in his right trigeminal territory. Magnetic resonance imaging showed a T2 hyperintensity of intrapontine trigeminal fibres and nucleus ( fig 1), without evidence of vascular conflicts with the trigeminal roo entry zone. A controlateral, smaller, symmetric lesion was also evident. Multiple sclerosis and Lyme disease were ruled out by clinical history and appropriate investigations. The patient had no vascular risk factors. Holter monitoring, neck vessels colour echo Doppler transthoracic echocardiographic examination, and brain MR angiography did no disclose alterations suggesting a possible ischaemic origin.

At operation, performed through a keyhole retromastoid craniectomy, the root entry zone of the nerve was found crossed by an "intratrigeminal" vein, which was electrocoagulated and divided. No other vascular contacts could be detected by careful exploration of the intracysternal tract of the nerve. The postoperative course was uneventful. Paroxysmal pain slowly faded away during

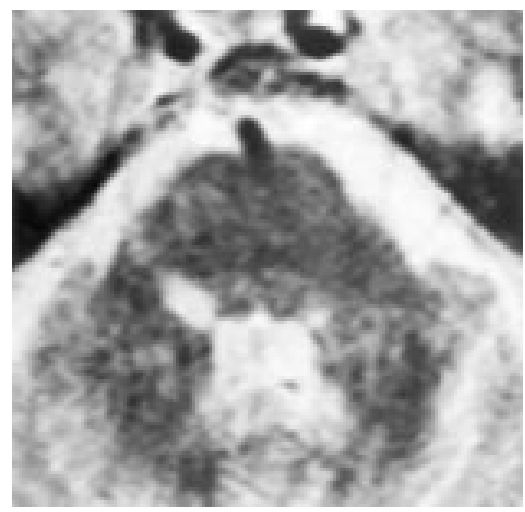

Figure 1 T2 weighted MRI showing hyperintensity of intrapontine trigeminal fibres. subsequent weeks. Carbamazepine could be completely withdrawn after 2 months.

This case is intriguing for two reasons: firstly, because we obtained an apparently paradoxical therapeutic answer in a case where microvascular decompression should have been generally contraindicated; secondly, because of the rarity of the T2 hyperintensity of intrapontine trigeminal fibres and nucleus in patients without multiple sclerosis

Although the intimate aetiopathogenetic mechanisms of trigeminal neuralgia stil remain unknown, peripheral lesions affecting the trigeminal nerve entry zone (tortuous vessels, meningiomas, schwannomas, aneurysms, artereovenous malfunctions, lipomas, epidermoid cysts, osteomas, etc) and multiple sclerosis are certainly involved in the mechanisms causing paroxysmal pain. Demyelination of trigeminal fibres at the level of trigeminal root entry zone in case of vascular cross compression and demyelination of intrapontine trigeminal fibres in case of multiple sclerosis (personal observation of MRI in more than $80 \%$ of cases) may result in ephaptic, abnormal transmission of impulses. A neurovascular conflict with focal demyelination at the root entry zone was surgically and pathologically confirmed in a patient with multiple sclerosis and trigeminal neuralgia by Lazar and Kirkpatrick. ${ }^{1}$ The anatomopathological evidence of demyelination of intrapontine trigeminal fibres in a patient with multiple sclerosis and trigeminal neuralgia was provided by Crooks and Miles.

Recent studies ${ }^{3}$ hypothesised that vascular compression (and possible consequent demyelination) of the trigeminal root and demyelination of intrapontine trigeminal fibres due to multiple sclerosis can coexist and perhaps cooperate in the genesis of painful attacks.

These findings led us to propose microvascular decompression to the patient rather than percutaneous lesive methods. At 2 year follow up the patient is still pain free, confirming the hypothesis that vascular compression and central demyelination can coexist and cooperate in provoking pain paroxysms. Hence, the classic distinction between the supposed "all central" mechanism for trigeminal neuralgia associated with multiple sclerosis and the "all peripheral" mechanism for the trigeminal neuralgia related to vascular compression should be overcome in favour of a unique (patients with trigeminal neuralgia and multiple sclerosis are included), mixed central-peripheral mechanism in which abnormal impulses coming from demyelinated axons (multiple sclerosis, vascular compression, and any other possible cause of demyelination along the central and the peripheral course of gasserian ganglion fibres) modulate the nuclear activity.

An alternative view of this case might be the one advocating the hypothesis that pain relief might be due to surgical damage to the trigeminal root. ${ }^{4}$ This view has been strongly challenged by the results of recent studies on sensory effects of microvascular decompression. ${ }^{5}$ In our patient, too, no evidence of sensory deficits could be found postoperatively.

Generally a minimum myelin damage, without any gross nerve hypofunction, is involved in the aetiopathogenesis of trigeminal neuralgia. In rare cases demyelination is so widespread along trigeminal fibres to be visualised by MRI and only patients with multiple sclerosis show the classic T2 hyperintensity along the intra-axial trigeminal pathways.

To the best of our knowledge this case is the third reported in which such a lesion was found in a patient without multiple sclerosis In the two previous $\operatorname{cases}^{67}$ a pontine ischaemia was supposed: in one, multiple cerebral ischaemic lesions with widespread cortical atrophy were found; in the other the pontine lesion was isolated, but an MR angiogram showed a focal stenosis of the vertebral artery. A few patients with small pontine infarcts were reported with trigeminal symptoms, but without paroxysmal pain.

In our case the MRI evident T2 hyperintensity of intrapontine trigeminal pathways was considered a demyelinating or an ischaemic lesion of unknown aetiology.

This case suggests that the presence of a pontine trigeminal lesion is not an absolute contraindication for microvascular decompression in cases of drug resistant typical trigeminal neuralgia.

P Ferroli

Department of Neurosurgery, Istituto Nazionale Neurologico C. Besta, Via Celoria 11, 20133,

Milan, Italy

A Franzini

Department of Neurosurgery

L Farina

Department of Neuroradiology

L La Mantia

Department of Neurology

G Broggi

Department of Neurosurgery

Correspondence to: Dr P Ferroli; ferrolipaolo@hotmail.com

\section{References}

1 Lazar ML, Kirkpatrick JB. Trigeminal neuralgia and multiple sclerosis: demonstration of the plague in an operative case. 1958;5:711-7

2 Crooks DA, Miles JN. Trigeminal neuralgia due to vascular compression in multiple sclerosis-post-mortem findings. Br J Neurology 1996;10:85-8.

3 Meaney JF, Watt JW, Eldridge PR, et al. Association between trigeminal neuralgia and multiple sclerosis: role of magnetic resonance imaging. J Neurol Neurosurg Psychiatry 1995;59:253-9

4 Adams CBT. Microvascular compression: an alternative view and hypothesis. I Neurosurg 1989:57:1-12.

5 Barker II FG, Jannetta PJ, Bissonette DJ, et al. Trigeminal numbness and tic relief after microvascular decompression for typical trigeminal neuralgia. Neurosurgery 1997; 40:39-45.

6 Balestrino $M$, Leandri M. Trigeminal neuralgia in pontine ischaemia. J Neurol Neurosurg Psychiatry 1997;62:297-8

7 Kim JS, Khang JH, Lee MC. Trigeminal neuralgia after pontine infarction. Neurology 1998:51:1511-12

\section{Reversal of tissue hypoxia by a} single intraventricular dose of sodium nitroprusside in a patient with severe medically refractory cerebral vasospasm after subarachnoid haemorrhage

A 29 year old man was referred to our department from a local hospital in November 2000 for treatment of acute subarachnoid haemorrhage. At examination, he presented with stupor and a Glasgow coma scale score of 7 and was intubated and artificially ventilated. Diagnostic angiography was performed the same day and demonstrated a ruptured arterior cerebral artery aneurysm. The complex configuration of the aneurysm precluded embolisation as a treatment option. Surgery was 


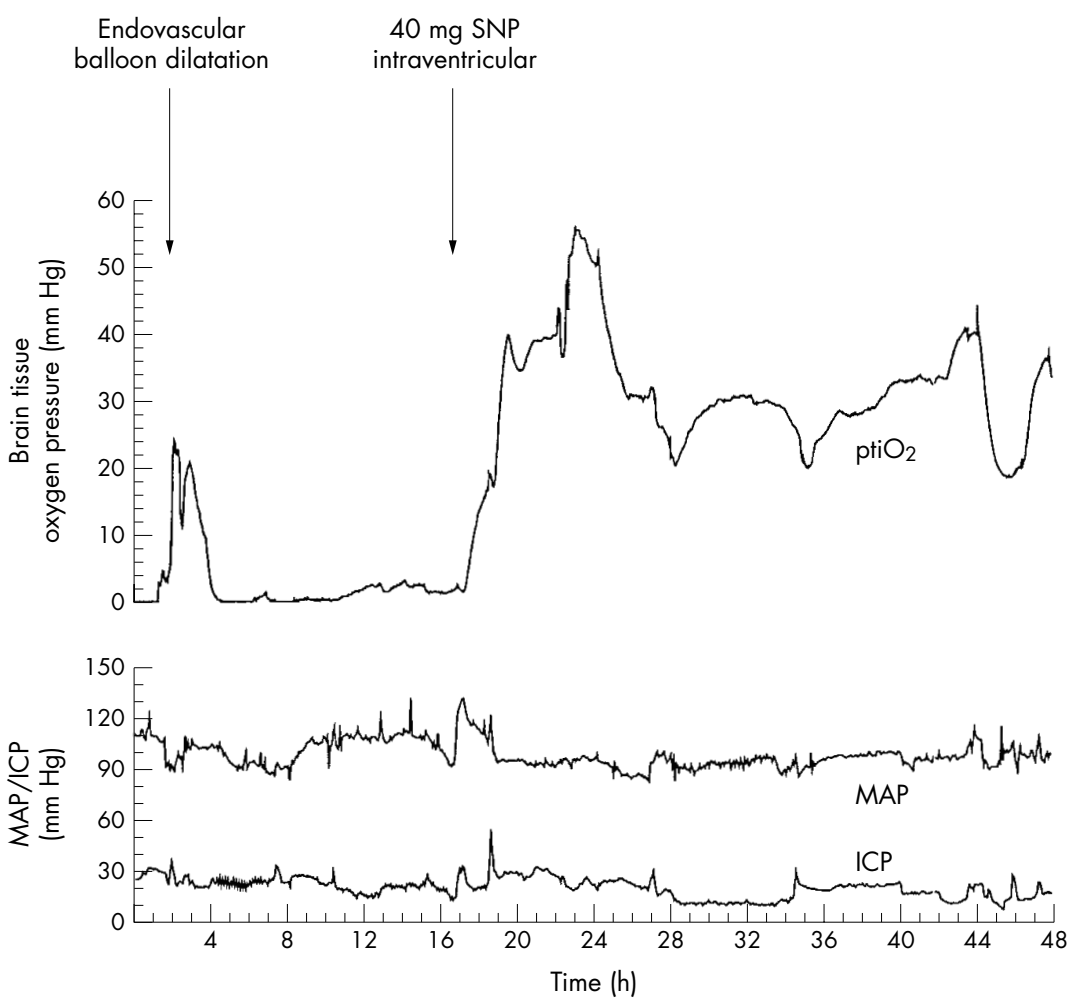

Figure 1 Left frontal brain tissue oxygen pressure $\left(\mathrm{ptiO}_{2}\right)$, mean arterial pressure (MAP), and intracranial pressure (ICP) in a patient with cerebral vasospasm after subarachnoid haemorrhage. Low ptiO ${ }_{2}$ values indicating critical brain hypoxia prompted emergency endovascular balloon dilatation of the vasospastic $\mathrm{Cl}$ segment of the left internal carotid artery, which only temporarily improved cerebral oxygenation. After intraventricular administration of a single dose of $40 \mathrm{mg}$ sodium nitroprusside, cerebral oxygenation improved permanently.

performed the next day and the aneurysm was successfully clipped. After surgery, the patient received the calcium channel blocker nimodipine intravenously at a dosage of 2 $\mathrm{mg} /$ hour and moderate hypervolaemic haemodilution using isotonic solutions to prevent vasospasm induced brain ischaemia. Transcranial Doppler flow velocities were less than $140 \mathrm{~cm} / \mathrm{s}$ from day 1 to 5 postoperatively. At day 6, Transcranial Doppler flow values increased up to $200 \mathrm{~cm} / \mathrm{s}$ indicating severe cerebral vasospasm. Cerebral angiography was performed demonstrating $80 \%$ vasospasm on the left internal carotid artery ( $\mathrm{Cl}$ segment). Because the patient remained in a medically induced coma, a Clark-type intraparenchymal brain tissue oxygen sensor was implanted in the left middle cerebral artery territory to monitor brain tissue oxygenation. Initial values showed tissue hypoxia (tissue oxygen pressure $<10 \mathrm{~mm} \mathrm{Hg}$ ), therefore, aggressive hypertensive hypervolaemic haemodilution therapy was initiated to improve cerebral circulation. After temporary improvement, the next day brain tissue oxygen pressure decreased below $5 \mathrm{~mm} \mathrm{Hg}$ and emergency endovasular balloon dilatation of the $\mathrm{Cl}$ segment of the internal carotid artery was performed. Again, tissue oxygen improved temporarily but critical tissue hypoxia developed 4 hours after balloon dilatation. Brain CT was performed and showed no signs of established cerebral infarction. At a brain tissue oxygen value of $2 \mathrm{~mm} \mathrm{Hg}$, a total dose of $40 \mathrm{mg}$ sodium nitroprusside was administered over a period of 30 minutes via the ventricular catheter. The dosage was chosen from an earlier clinical report. ${ }^{1}$ Seventy minutes after sodium nitroprusside administration, brain tissue oxygen increased continuously and persisted at normal values of $>15 \mathrm{~mm} \mathrm{Hg}$ for the next days (fig 1). The clinical course was further complicated by bilateral pneumonia and acute respiratory distress syndrome. Follow up CT showed no major infarction but an atypical left frontal $2 \times 2 \times 2 \mathrm{~cm}$ hypodense white matter lesion. The patient was discharged at Rankin grade 5 and slightly improved to Rankin grade 4 after 6 months.

Cerebral vasospasm is the most significant surviving subarachnoid haemorrhage long enough to reach medical care. Using the data from the vehicle group of the recent randomised, double blind, vehicle controlled trials on tirilazad mesylate, ${ }^{2}$ symptomatic vasospasm occurred in 33\%-38\% and cerebral infarctions from vasospasm were found in $10 \%-13 \%$ of all patients after subarachnoid haemorrhage. Prevention and treatment of cerebral vasospasm were achieved despite the use of nimodipine, hypertensive hypervolaemic hemodilutive therapy, angioplasty and intra-arterial papaverine administration. So far, there is no other established treatment modality that could be offered to those patients with severe vasospasm, decreasing blood flow, and impending cerebral infarction.

The reduction in the concentration of nitric oxide is one purported mechanism of delayed vasospasm after subarachnoid haemorrhage. Evidence in support of a causative role for nitric oxide includes the disappearance of nitric oxide synthase from the adventitia of vessels in spasm, the destruction of nitric oxide by haemoglobin released from the clot cause of morbidity and mortality in patients into the subarachnoid space, and reversal of vasospasm by intracarotid nitric oxide.

Recently, Thomas et al have suggested intraventricularly administered sodium nitroprusside as a treatment for severe medically refractory vasospasm after subarachnoid haemorrhage. ${ }^{13}$ After intraventricular administration, sodium nitroprusside is supposed to attenuate vasospasm by releasing nitric oxide on the abluminal side of the basal cerebral vessels.

Apart from the original description of the clinical and angiographic effects there is only one report that repeated intraventricular sodium nitroprusside administration indeed may improve crirically reduced cerebral oxygenation and blood flow. ${ }^{4}$ Our data demonstrate for the first time that despite the short half life of sodium nitroprusside a single intraventricular dose may lead to permanent improvement of reduced cerebral oxygenation in the territory of a severely vasospastic artery. Our finding supports the concept that nitric oxide is playing a critical part in the pathophysiology of cerebral vasospasm after subarachnoid haemorrhage and that intraventricular sodium nitroprusside may offer a a novel therapeutic option in patients with otherwise medically refractory vasospasm and impending cerebral infarction.

A Raabe, H Vatter, M Zimmermann,

V Seifert

Department of Neurosurgery, Neurology and Neurosurgery Centre, Johann Wolfgang Goethe University, Schleusenweg 2-16, 60528 Frankfurt am Main, Germany

Correspondence to: Dr A Raabe; A.Raabe@em.uni-frankfurt.de

\section{References}

1 Thomas JE, Rosenwasser RH. Reversal of severe cerebral vasospasm in three patients after aneurysmal subarachnoid hemorrhage: initial observations regarding the use of intraventricular sodium nitroprusside in humans. Neurosurgery 1999;44:48-57.

2 Haley EC Jr, Kassell NF, Apperson-Hansen $C$, et al. A randomized, double-blind vehicle-controlled trial of tirilazad mesylate in patients with aneurysmal subarachnoid hemorrhage: a cooperative study in North America. J Neurosurg 1997;86:467-74.

3 Thomas JE, Rosenwasser RH, Armonda RA, et al. Safety of intrathecal sodium nitroprusside for the treatment and prevention of refractory cerebral vasospasm and ischemia in humans. Stroke 1999;30:1409-16.

4 Vajkoczy P, Hubner U, Horn P, et al. Intrathecal sodium nitroprusside improves cerebral blood flow and oxygenation in refractory cerebral vasospasm and ischemia in humans [letter]. Stroke 2000;31:1195-7.

\section{Transcranial magnetic stimulation alleviates truncal ataxia in spinocerebellar degeneration}

Spinocerebellar degeneration is an inherited or acquired neurodegenerative disorder characterised by steadily progressive cerebellar ataxia, dysarthria, and gait disturbance. These symptoms restrict daily activities. However, no satisfactory therapy has been established. Transcranial magnetic stimulation (TMS), originally introduced to the medical field to evaluate the function of the CNS, is recently becoming a therapeutic tool for neuropsychiatric disorders, such as major depression ${ }^{1}$ and Parkinson's disease. ${ }^{2}$ We also reported the efficacy of TMS for inherited spinocerebellar 
Table 1 Age, disease duration, and disease severity of patients enrolled in this study and the results of comparisons between active stimulation and sham stimulation, and between cerebellar-type SCD and OPCA type SCD receiving active stimulation

\begin{tabular}{|c|c|c|c|c|c|c|}
\hline & Active & Sham & p Value & Active cerebellar & Active OPCA & pValueł \\
\hline Age (y) & $56.31(1.96)$ & $58.83(1.47)$ & 0.32 & $62.00(2.03)$ & $54.48(2.80$ & 0.08 \\
\hline Disease durarion $(y)$ & $7.73(0.71)$ & $6.67(0.82)$ & 0.33 & $8.28(1.27)$ & $5.52(0.91)$ & 0.10 \\
\hline Disease types (N) (cerebellar:OPCA) & $17: 22$ & $16: 19$ & 0.85 & & & \\
\hline \multicolumn{7}{|l|}{$10 \mathrm{~m}$ time $(\mathrm{s}):$} \\
\hline $\begin{array}{l}\text { Before TMS } \\
\text { After TMS }\end{array}$ & $14.37(1.80)$ & $13.78(1.23)$ & 0.79 & $13.74(2.92)$ & $16.24(2.26)$ & 0.23 \\
\hline \multirow{2}{*}{\multicolumn{4}{|c|}{10 m steps: }} & $8.11(0.49)$ & $12.16(1.21)$ & $<0.05$ \\
\hline Before TMS & $23.88(2.19)$ & $25.19(1.71)$ & 0.64 & & & 0.28 \\
\hline After TMS & $19.47(1.19)$ & $23.49(1.69)^{*}$ & $<0.05$ & $19.97(1.89)$ & $19.39(1.55$ & 0.40 \\
\hline \multicolumn{7}{|l|}{ Tandem steps: } \\
\hline Before TMS & $1.24(0.37)$ & $2.26(0.67)$ & 0.19 & $2.39(0.77)$ & $1.05(0.49)$ & 0.20 \\
\hline After TMS & $7.92(2.12)$ & 3.25 (1.38) & $<0.005$ & $10.72(3.52)$ & $4.81(1.87)$ & $<0.05$ \\
\hline \multicolumn{7}{|l|}{ Standing capacities: } \\
\hline Before TMS & $2.36(0.18)$ & $2.43(0.21)$ & 0.72 & $2.11(0.34)$ & $2.48(0.18)$ & 0.17 \\
\hline After TMS & $1.64(0.11)$ & $2.11(0.17)^{*}$ & $<0.05$ & $1.23(0.16)$ & $1.86(0.13)$ & $<0.01$ \\
\hline \multicolumn{7}{|l|}{ Walking capacities: } \\
\hline Before TMS & $2.21(0.12)$ & $2.11(0.20)$ & 0.64 & $2.00(0.34)$ & $2.19(0.13)$ & 0.41 \\
\hline Affer TMS & $1.67(0.13)$ & $2.07(0.20)$ & 0.09 & $1.23(0.16)$ & $1.62(0.18)$ & 0.51 \\
\hline
\end{tabular}

Values are shown as mean (SEM).

$10 \mathrm{~m}$ time, $10 \mathrm{~m}$ steps, Tandem steps are indicated by the same abbreviations used in the text.

* $p<0.05$ compared with each value before TMS in sham stimulation group; $† p$ Values shown in this table were analyzed between active and sham stimulation groups; $\ddagger$ V Values shown in this table were analyzed between cerebellar type and OPCA type of active stimulation group.

degeneration. ${ }^{3}$ We now report here a placebo controlled trial of TMS over the cerebellum for patients with spinocerebellar degeneration.

Seventy four patients with spinocerebellar degeneration gave written informed consent to participate in this study, which was approved by the ethics committee of Tohoku University. No patient had a history of seizures or any abnormalities established by EEG. They also had no orthopaedic problems. Thirty nine patients, aged from 27 to 76 years (19 men and 20 women), were assigned to active stimulation, and the other 35 patients, aged from 38 to 76 years $(25$ men and 11 women), were assigned to sham stimulation. They were divided based on the date when they were admitted to our hospitals. The age, disease duration (unpaired $t$ test), disease type ( $\chi^{2}$ test), and disease severity (unpaired $t$ test) were matched between the two groups. Disease types were divided into two groups, cerebellar type (sporadic and hereditary cortical cerebellar atrophy including spinocerebellar atrophy (SCA) 6) and olivopontocerebellar atrophy (OPCA) type (sporadic OPCA, SCAl, SCA3, etc). Transcranial magnetic stimulation over the cerebellum was administered at almost the same time in the evening once a day for 21 consecutive days. A Magstim 200 (Magstim, Wales, UK), a transcranial magnetic stimulator with a $14 \mathrm{~cm}$ circular coil, was used. The stimulus coil was placed tangentially (active stimulation) or vertically (sham stimulation) over the scalp and centred on the inion, $4 \mathrm{~cm}$ lateral to the right of the inion, and $4 \mathrm{~cm}$ lateral to the left of the inion. The site order of delivery was always the same, first the right, then the centre, and then the left repeatedly 10 times in the same order. Ten pulses (the interpulse interval was about 6 seconds, which depended on the time that the Magstim 200 needed to be fully charged; five were counter clockwise and five were clockwise) were delivered on each region. The duration of each stimulus pulse was $0.1 \mathrm{~ms}$. The stimulator output was adjusted to $100 \%$ of the maximum output capacity, which was about 2.5 times the motor threshold of the participants. We measured the motor thresh old by stimulating the hand area of the left cortex using a $14 \mathrm{~cm}$ circular coil and recording the motor evoked potentials from the right first dorsal interosseous muscle while the hands were relaxed. The average threshold was 39.4 (SD 3.8$) \%$.

We evaluated their truncal ataxia according to the time required for a $10 \mathrm{~m}$ walk $(10 \mathrm{~m}$ time), the number of steps for a $10 \mathrm{~m}$ walk ( 10 $\mathrm{m}$ steps), and the number of practicable steps on a walk with the feet in the tandem position (tandem steps). Standing capacities ( 0 , able to stand on one foot more than 15 seconds; 1 , able to stand with the feet in the tandem position; 2, able to stand with the feet together; 3, able to stand with the heels together; 4 , able to stand with the feet less than $10 \mathrm{~cm}$ apart; 5, able to stand with feet more than $10 \mathrm{~cm}$ apart; 6 , unable to stand without support) and walking capacities (0, normal; 1 , almost normal but unable to run; 2 , able to walk without support, but clearly abnormal; 3 , able to walk without support but with considerable staggering; 4 , able to walk with a handrail; 5 , able to walk with considerable support; 6, unable to walk, even with accompanying person) were also evaluated The evaluation was done by the patients' physicians, who did not participate in this study. We measured the regional brain blood flow by consecutive single photon emission computed tomography (SPECT) using technetium-99m ethyl cystinate dimer before and after the 3 week TMS trial in 28 patients in the active stimulation group. We put the regions of interest on the bilateral frontal lobes, temporal lobes, occipital lobes, putamina, cerebellar hemispheres and pontine base. Quantitative blood flow in each region of interest was calculated from qualitative axial SPECT images by the application of Patlak plot graphical analysis. ${ }^{4}$ The examiners were blind to the manner of TMS.

All participants completed the 3 week TMS trial without any adverse effects. One patient was fearful and needed a reduction in the stimulus intensity to $80 \%$ of the maximum output. Before the TMS trial, there were no differences in $10 \mathrm{~m}$ time, $10 \mathrm{~m}$ steps, tandem steps, standing capacities, and walking capacities in both groups (Mann-Whitney $U$ test). Comparing between active and sham stimulation after 3 week TMS, active stimulation was significantly more effective in $10 \mathrm{~m}$ time $(\mathrm{p}<0.05), 10 \mathrm{~m}$ steps $(\mathrm{p}<0.05)$, tandem steps $(\mathrm{p}<0.005)$, and standing capacities $(\mathrm{p}<0.05)$ (Mann-Whitney $U$ test, table 1$)$. However, $10 \mathrm{~m}$ time $(\mathrm{p}<0.05), 10 \mathrm{~m}$ steps $(\mathrm{p}<0.05)$, and standing capacities $(\mathrm{p}<0.05)$ were significantly improved in the sham stimulation group (Wilcoxon signed rank test, *table 1). The cerebellar type of spinocerebellar degeneration was significantly more sensitive to TMS than the OPCA type in $10 \mathrm{~m}$ time $(\mathrm{p}<0.05)$, tandem steps $(\mathrm{p}<0.05)$, and standing capacities $(\mathrm{p}<0.01)$ (Mann-Whitney $U$ test, table 1). After the 3 week TMS trial, the mean regional brain blood flow significantly increased in the cerebellum and pons from 52.52 (SEM 2.12) $\mathrm{ml} / 100 \mathrm{~g} / \mathrm{min}$ to 58.54 (SEM 1.89 ) $\mathrm{ml} / \mathrm{l} 00 \mathrm{~g} / \mathrm{min}(\mathrm{p}<0.005)$, and from 34.38 (SEM 2.21 ) $\mathrm{ml} / 100 \mathrm{~g} / \mathrm{min}$ to 39.68 (SEM 1.34) $\mathrm{ml} / 100 \mathrm{~g} / \mathrm{min}(\mathrm{p}<0.05)$, respectively (paired $t$ test). The regional brain blood flow in the cerebral cortices did not show any change.

In this study, we found a significant alleviation of truncal ataxia in patients with spinocerebellar degeneration treated by active TMS Although placebo or training effects were demonstrated in $10 \mathrm{~m}$ time, $10 \mathrm{~m}$ steps, and standing capacities $\left({ }^{*}\right.$ table 1$)$, the effects of active stimulation were far beyond those of sham stimulation. With our method, active stimulation evoked the contraction of nuchal and shoulder muscles. However, sham stimulation produced the same noise as that of active stimulation, and some scalp sensation. Patients did not know the difference between active and sham stimulation because no patients had experienced active stimulation previously. Therefore, patients having sham stimulation did not notice that they were receiving inactive stimulation. Our results showed that the disease type was important for the effect of TMS. We matched not only the age, disease duration, and disease severity of both groups but also the type of disease. We considered this study to be placebo controlled. Therefore, TMS over the cerebellum actually alleviates truncal ataxia in patients with spinocerebellar degeneration. 
Although we do not yet know the mechanism by which TMS works, we are very much interested in the increase in cerebellar blood flow after TMS. This finding suggests that TMS over the cerebellum may activate the decreased cerebellar function. This effect may be caused by direct stimulation to the cerebellum as we used the maximum strength of stimulation at 2.5 times the motor threshold. Another possibility is that sensory input from the contracted muscles by TMS might occur.

After the TMS trial we continued TMS in some patients. Patients receiving TMS once or twice a week kept their improved condition at least for 6 months, but patients receiving TMS every 2 weeks returned to their baseline in 2 weeks. Therefore, we think that the effects of TMS with our method are maintained for about 1 week. We conclude that TMS over the cerebellum is a promising treatment for spinocerebellar degeneration.

\section{Acknowledgement}

This work was financially supported by the Magnetic Health Science Foundation.

Y Shiga, T Tsuda, Y Itoyama

Department of Neurology, Tohoku University Schoo of Medicine, 1-1 Seiryo-machi, Aoba-ku, Senda 980-8574, Japan

H Shimizu

Department of Neurology, Yamagata Nationa Hospital, Japan

K-I Miyazawa

Department of Neurology, Kohnan Hospital, Japan

K Jin

Department of Neurology, Miyagi National Hospital, Japan

T Yamazaki

Department of Radiology, Tohoku University Schoo of Medicine, Japan

Correspondence to: $\operatorname{Dr}$ Y Shiga yshiga@neurol.med.tohoku.ac.jp

\section{References}

1 Pascual-Leone A, Rubio B, Pallardo F, et al. Rapid rate transcranial magnetic stimulation of the left dorsolateral prefrontal cortex in drug-resistant depression. Lancet 1996:348:283-7.

2 Mally J, Stone TW. Improvement in parkinsonian symptoms after repetitive transcranial magnetic stimulation. J Neurol Sci 1999; 162:179-84.

3 Shimizu H, Tsuda T, Shiga $Y$, et al. Therapeutic efficacy of transcranial magnetic stimulation for hereditary spinocerebellar degeneration. Tohoku J Exp Med 1999;189:203-1 1.

4 Matsuda H, Yagishita A, Tsuji S, et al. A quantitative approach to technetium- $99 \mathrm{~m}$ ethyl cystinate dimer: a comparison with technetium-99m hexamethyl propylene amine oxine. Eur I Nucl Med 1995:22:633-7.

\section{Agraphia in Kanji after a contusional haemorrhage in the left temporo-occipital lobe}

The Japanese language has two different writing systems, Kana (Japanese syllabograms) and Kanji (Japanese ideograms), and Japanese sentences usually consist of combinations of both. Recently, there has been speculation that different pathways are involved in Kanji and Kana reading and writing. It has been suggested that the processing of Kanji and Kana involves different intrahemispheric mechanisms, as judged mainly through studies on patients with

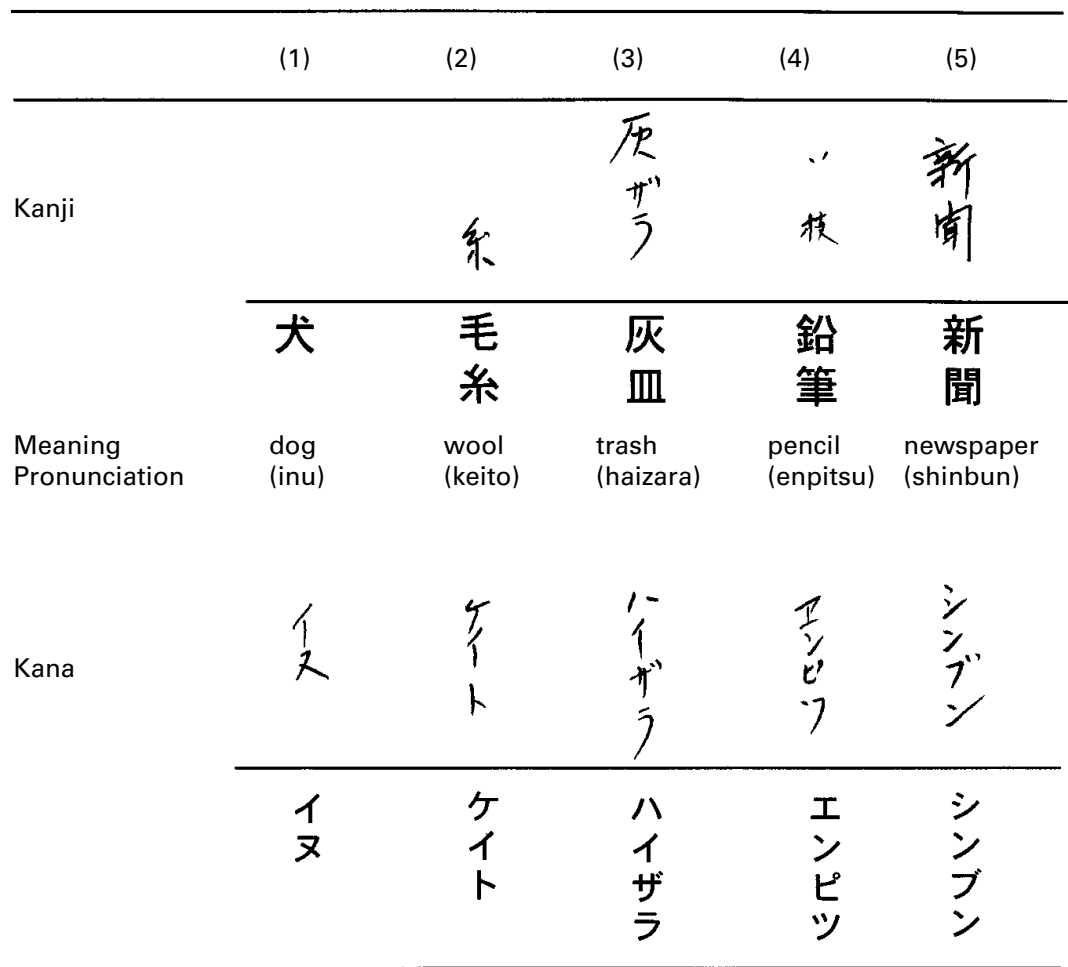

Figure 1 Examples of dictation. This patient showed intact Kana writing and difficulties in Kanji writing: 1, no response; 2, partial completion; 3, partial substitution; 4, neographism; 5, correct.

alexia and agraphia. ${ }^{2}$ Wernicke's area and its surrounding left middle temporal lobe may play the most important part in Kanji reading when visual information is transmitted by any pathway. We recently had the opportunity to examine a patient who had mild transient aphasia and persistent agraphia of Kanji after a discrete contusional haemorrhage in the left temporo-occipital lobe.

A 66 year old right handed man (pensioner) with 12 years of school education was involved in a traffic accident and sustained a closed head injury. He was admitted to our hospital the next day. He had no history of neurological problems. His parents, brothers, and sisters are all right handed. On examination, he was alert and cooperative. Neurological examination disclosed no motor or sensory disturbance.

Formal language assessment was undertaken 3 days after injury using the standard language test for aphasia (SLTA). His spontaneous speech was fluent. He had no difficulty initiating speech, articulated normally, and did not have logorrhoea. Echolalia was not seen and phonological structure was clear. Object naming was moderately impaired, with considerable paraphasia. Word fluency (animal naming) was 3/min. Repetition was excellent. Reading aloud was good; however, writing of both Kanji and Kana was impaired, and considerable paragraphia was evident.

In addition, he demonstrated constructional apraxia using figure copying of a three dimensional cube but not oral, ideomotor, or ideational apraxia. There were no signs of motor impersistence or unilateral spatial neglect. The score on Raven's coloured progressive matrices was 26/36.

Brain CT on admission showed an area of high density consistent with contusional haemorrhage in the left temporo-occipital lobe. Brain MRI 5 days after admission showed contusional haemorrhage in the left temporo-occipital lobe involving the posterior part of the temporal cortex and the adjacent white matter. There were no abnormalities in the left angular gyrus.

Aphasia was gradually resolved, his scores on SLTA 8 weeks after the onset becoming normal. Despite this, his writing ability remained clearly disturbed. We asked him to read and write on dictation 221 Kanji and 76 Kana, all of which are taught in the first 2 years of primary school in Japan. He was unable to write 83 of the 221 Kanji (37.6\%), which he reported that he knew but could not recall. He tended to have difficulty with Kanji characters, which were more complex and had been learnt later (fig 1). Error types included partial substitution (two), partial completion (seven), neographism (29), and no response (45). On the other hand, he made no mistakes in reading Kanji or in reading and writing Kana letters. On the revised Wechsler adult intelligence scale (WAIS-R), his verbal intelligence quotient (IQ) was 105 and his performance IQ was 101, with an overall IQ of 103. Brain MRI 3 months after admission showed a subcortical lesion in the left temporo-occipital lobe (fig 2).

Although this patient experienced aphasia immediately after cerebral contusion, the aphasia resolved over a subsequent 8 week period. He was left with an isolated disturbance of Kanji writing. In the absence of any dementia, aphasia, or disturbance of consciousness, his condition was classified as one of pure agraphia.

Soma et $a l^{2}$ described "pure agraphia of Kanji" in three patients, who had a lesion in the left posterior temporal area extending to the angular gyrus on CT. Although they 


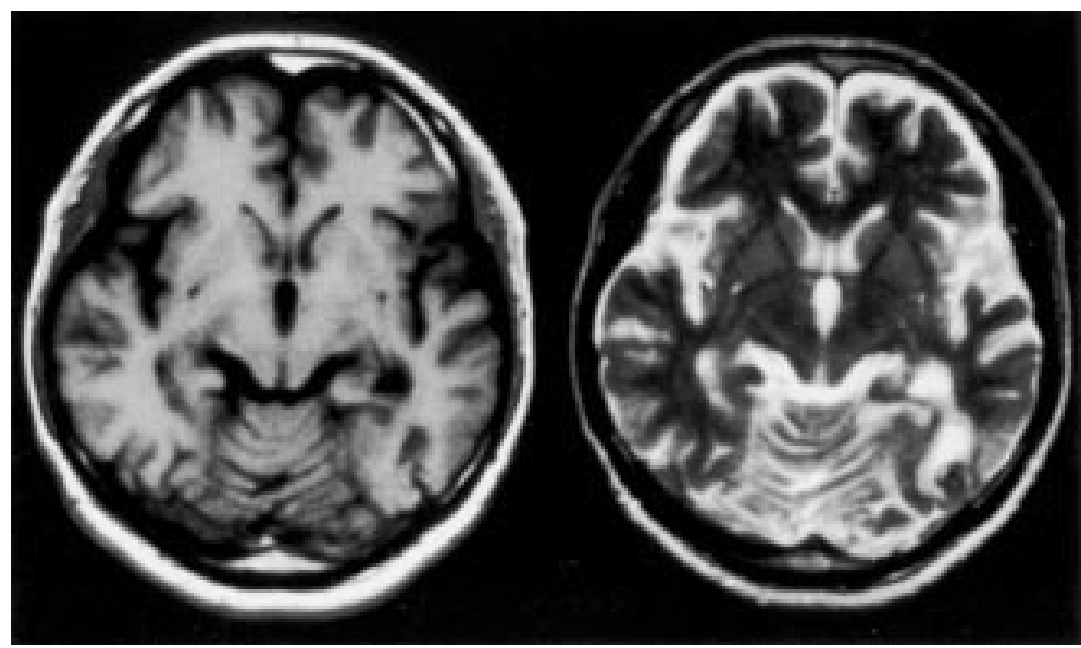

Figure 2 Brain MRI 3 months after admission showed the subcortical lesions in the left temporo-occipital lobe.

exhibited amnestic aphasia, alexia, and agraphia in the acute phase, disturbances other than agraphia of Kanji disappeared in a few months. Yokota et al ${ }^{3}$ also reported a case with pure agraphia of Kanji, and suggested that the process of writing Kanji involves a different pathway from that which mediates Kanji reading in the left temporal lobe.

Iwata ${ }^{1}$ proposed a hypothetical neuronal mechanism in the writing of Kanji and Kana as follows: the Broca's and motor association areas are the final coordination centres, but the angular gyrus plays an important part in sending the graphic information to the motor areas. However, spontaneous writing and responses to dictation are usually initiated by Wernicke's area, which gives rise to two different pathways to the angular gyrus. One is the auditory somaesthetic association pathway leading directly from Wernicke's area to the angular gyrus; Kana writing mainly depends on the intactness of this route. The second pathway is from Wernicke's area to the occipital lobe by way of the posterioinferior temporal area. This is the pathway involved in selecting the correct Kanji graphemes according to the meaning of the word, and thus recalled visual engrams of letters are sent to the angular gyrus.

Our patient demonstrated that initial amnestic aphasia, and agraphia of Kanji were associated with a lesion in the left temporal lobe. Because the posterior temporal region is located in close proximity to the angular gyrus and Wernicke's area, the pathological process in the first area affects the second two regions in its acute phase. We conclude that the persistent symptom of pure agraphia for Kanji in this study was caused by the left posterio temporal lesion which disconnected the pathway for Kanji writing selectively.

S Maeshima, A Ueyoshi, T Matsumoto Department of Physical Medicine and Rehabilitation, Wakayama Medical University, Wakayama, Japan

R Okita, H Yamaga, F Ozaki, H Moriwaki Division of Neurological Surgery, Hidaka General Hospital, Wakayama, Japan

P Roger

School of Communication Sciences and Disorders, University of Sydney, Australia
Correspondence to: Dr S Maeshima; maeshima@wakayama-med.ac.jp

\section{References}

1 Iwata M. Kanji versus Kana. Neuropsychological correlates of the Japanese writing system. Trans Neurosci 1984:7:290-3

2 Soma Y, Sugishita M, Kitamura K, et al. Lexical agraphia in the Japanese language. Pure agraphia for Kanii due to left posterioinferior temporal lesions. Brain 1989:112:1549-61

3 Yokota T, Ishiai S, Furukawa T, et al. Pure agraphia of kanji due to thrombosis of the Labbe vein. J Neurol Neurosurg Psychiatry 1990;53:335-8.

\section{Successful autologous stem cell transplantation in a patient with chronic inflammatory demyelinating polyneuropathy}

A patient with chronic inflammatory demyelinating polyneuropathy is reported on who never had spontaneous remissions for 10 years, but who is in remission for 2 years after an autologous stem cell transplantation (ASCT). Before ASCT he needed at least $20 \mathrm{mg}$ prednisone/day and in addition intravenous immunoglobulin (IVIg) treatment at regular intervals. An ASCT was considered in this patient because of serious side effects of immunosuppressive treatment.

Chronic inflammatory demyelinating polyneuropathy (CIDP) is characterised by weakness with sensory impairments in the arms and legs. The reflexes in the arms and legs disappear. The onset is insidious. There may be progressive deterioration or a course with remissions and exacerbations. Routine blood examination is usually normal. Protein in CSF is increased in most patients. Electrophysiological studies may show slow nerve conduction velocities, conduction blocks, or dispersion. ${ }^{1} \quad$ Inflammatory demyelinating polyneuropathy is considered to be an autoimmune disease which is supported by the presence of inflammatory cells in sural nerve biopsies and the beneficial response to immunosuppressive treatment in most patients. ${ }^{2} \mathrm{We}$ describe a patient with CIDP who has had this disease for 10 years without spontaneous remissions. He responded to immunosuppressive therapy, but needed high doses and had severe side effects. Therefore, we decided to try to induce a long lasting remission by autologous stem cell transplantation (ASCT).

This patient was 38 years old when, in 1988 , he began to have tingling and numbness in his fingers. The sensory symptoms progressed to his arms and legs, followed by weakness. Initially his symptoms were attributed to too much stress but at the end of 1990 when weakness had worsened to such an exten that he was no longer independent in his daily activities, it was decided that it was time for a neurological evaluation. At that time he had weakness of his arms and legs, MRC grade 4. There was atrophy of his intrinsic hand muscles and fasciculations were seen in the muscles of his arms. Except for diminished knee reflexes, there was areflexia. He had numbness of his arms and legs in a glove and stocking distribution. Electrophysiological studies showed slowed nerve conduction velocities of the median, ulnar, tibial posterior, and peroneal nerves in the range of $15-25 \mathrm{~m} / \mathrm{s}$ with prolonged distal latencies and $\mathrm{F}$ wave responses. The distal compound muscle action potentials were small. Few muscles had signs of denervation. Blood examination was unremarkable and CSF total protein was $0.67 \mathrm{~g} / \mathrm{l}$ without cells. Sural nerve biopsy was consistent with CIDP, showing inflammatory cells and demyelination.

In 1990 he was started on $60 \mathrm{mg} /$ day prednisone. After 2 weeks improvement began. This improvement continued to normal strength leaving only slight numbness in his fingers. During tapering off of the prednisone dose he had mood disturbances at every change of dose. Repeatedly he had a relapse a doses lower than $20 \mathrm{mg} /$ day. Subsequently we tried to replace prednisone by $150 \mathrm{mg} /$ day azathioprine but after 2 years of treatment with azathioprine it was still not possible to decrease the dose of prednisone to less than $20 \mathrm{mg} /$ day. Azathioprine was replaced by methotrexate (7.5 mg/week) for 6 months without a beneficial response. Thereafter he was treated with IVIg. After two cycles of $30 \mathrm{~g} /$ day IVIg for 5 days he needed infusions every other week to maintain his improved condition. We tried to prolong these intervals by adding prednisone. With $20 \mathrm{mg} /$ day prednisone he remained free of relapses for about 4 months. However, these intervals became gradually shorter; he developed arthralgias after administration of IVIg, and had repeated gastric pain. After 8 years of immunosuppressive treatment, without spontaneous remissions, we discussed the possibility of autologous stem cell transplantation (ASCT).

In May 1998 peripheral blood stem cells were harvested by leukopheresis after mobilisation with cyclophosphamide $\left(4 \mathrm{~g} / \mathrm{m}^{2}\right)$ and granulocyte colony stimulating factor (G-CSF; 5 $\mu \mathrm{g} / \mathrm{kg}$ ). CD 34+ cells were positively selected by immunomagnetic beads (Clinimax ${ }^{\circledR}$, purity 98.7\%) and cryopreserved. Before this procedure the patient had had IVIg every other week together with $20 \mathrm{mg} /$ day prednisone. With this regular IVIg treatment he was in an optimal condition: he had no weakness and only mild sensory impairment of his fingers. After the mobilisation procedure, prednisone could be tapered off to $8 \mathrm{mg} / \mathrm{day}$. Even without IVIg he remained in this improved condition for 5 months before he relapsed. Weakness in the arms and legs returned together with sensory impairment. Electrophysiological studies were repeated. We decided firstly to improve his neurological condition by IVIg treatment before myeloablative treatment was started. This treatment resulted in considerable improvement; only mild sensory impairment of the fingers remained. 
In March 1999 he was treated with BEAM myeloablative chemotherapy $\left(300 \mathrm{mg} / \mathrm{m}^{2}\right.$ BCNU on day $-6,200 \mathrm{mg} / \mathrm{m}^{2} /$ day etoposide on days $-5,-4,-3$, and $-2,200 \mathrm{mg} / \mathrm{m}^{2} /$ day cytosine arabinoside on days $-5,-4,-3$, and -2 , and $140 \mathrm{mg} / \mathrm{m}^{2} /$ day melphalan on day-1) followed by stem cell re-infusion at day $0\left(11.2 \times 10^{6} \mathrm{CD} 34+\right.$ cells $\left./ \mathrm{kg}\right)$. Afte re-infusion recovery of peripheral blood cells occurred within 16 days. Prednisone was tapered off but doses lower than $5 \mathrm{mg}$ were accompanied by complaints of fatigue probably due to adrenal insufficiency. Two years after stem cell re-infusion, he is still free of relapses with $5 \mathrm{mg}$ /day prednisone and without IVI g treatment. He works full time and the only neurological signs consist of mild numbness of his fingertips. Electrophysiological studies were repeated 1 year after reinfusion and compared with the latest study performed when he had relapsed after the mobilisation phase. For the right median nerve, the distal CMAP improved from $2.1 \mathrm{mV}$ to $7.3 \mathrm{mV}$, the distal latency from 14.6 to 12.3 $\mathrm{ms}$, and the motor nerve conduction velocity from 12 to $24 \mathrm{~m} / \mathrm{s}$.

This is the first report of autologous CD 34+ stem cell transplantation in a patient with CIDP. Recently, Marmont reviewed the results of autologous stem cell transplantation (ASCT) in autoimmune diseases. ${ }^{3}$ He discussed a group of 143 patients, including patients with multiple sclerosis $(n=51)$, systemic sclerosis, $(n=30)$, rheumatoid arthritis $(n=15)$, juvenile chronic arthritis $(n=18)$, systemic lupus erythematosus $(n=12)$, vasculitis $(n=4)$, other connective tissue disease $(n=4)$, and various autoimmune haematoogical disorders $(n=9)$. The treatment of these patients was variable: some had trans plantation of bone marrow stem cells, other had mobilised peripheral blood haematopoi etic stem cell transplantation. The graft wa unmanipulated in 36 patients and was $\mathrm{T}$ cell depleted in the remaining patients. The high est relapse rate at 1 year was seen in juvenile chronic arthritis (50\%) whereas only $10 \%$ of patients with multiple sclerosis had relapsed. The transplant related mortality at 2 years post-transplantation was 6\%. Multiple sclerosis has become the autoimmune disease which is most often treated by ASCT.

Marmont discussed a group of 24 patients with multiple sclerosis with a progressive course who were treated similarly to the patient with CIDP in this report. The patients with multiple sclerosis were conditioned with the BEAM regimen; the mobilisation phase consisted of cyclophosphamide followed by granulocyte colony stimulating factor and finally CD 34+ cells were cryopreserved. The only difference was that our patient was not treated with antithymocyte globulin. In the group of 24 patients with multiple sclerosis, one patient died of aspergillosis in the post-transplant period. The other 23 patient had no severe transplant related morbidity. Improvement in disability was seen in 10 patients, stabilisation in another 10

The question is how ASCT is capable of eradicating autoimmunity. Remissions after allogeneic stem cell transplantation are easier to explain, as a "diseased" immunological and stem cell compartment is replaced with one that is normal and likely to develop tolerance. However, allogeneic transplantations are ac companied by high mortality figures and are therefore not considered in non-lethal autoimmune diseases.

Autologous transplantations can better be described as rescue therapy. High doses of cytotoxic therapy as is given in ASCT are also myeloablative and are only possible with stem cell rescue treatment. It might be that the effects of this treatment on autoimmune diseases can be attributed completely to the high levels of cytotoxic treatment. ${ }^{4}$ However, development of tolerance by "re-educated" lymphocytes from the purified CD $34+$ cells has not been ruled out.

We certainly do not advise the routine use of ASCT in patients with CIDP. Most patients have an excellent response to immunomodulating therapy without serious side effects. Moreover, after several years of treatment many patients reach long lasting remissions. However, if high doses of immunosuppressive therapy are necessary for long periods and are poorly tolerated, ASCT with a well tolerated conditioning regimen may be considered.

$M$ Vermeulen

Department of Neurology (H2-220), Academic Medical Center, University of Amsterdam, PO Box 22700, 1100 DE Amsterdam, The Netherlands

M H Van Oers

Department of Haematology

Correspondence to: $\operatorname{Dr} M$ Vermeulen m.vermeulen@amc.uva.n

\section{References}

1 Mc Combe PA, Pollard JD, McLeod JG. Chronic inflammatory demyelinating polyradiculopathy. A clinical and electrophysiological study of 92 cases. Brain 1987:110:1617-30.

2 Hadden RD, Hughes RA, Treatment of immune-mediated inflammatory neuropathies. Curr Opin Neurol 1999;12:573-9.

3 Marmont AM. New horizons in the treatment f autoimmune diseases: immuno ablation and tem cell transplantation. Ann Rev Med 2000;51:115-34

4 Nash RA. Prospects of stem cell ransplantation in autoimmune diseases. J Clin Immunol 2000;20:38-45.

\section{CORRESPONDENCE}

\section{Unusual presentation of a primary spinal lymphoma}

Wilkening et al described the unusual history of a formerly healthy immunocompetent white woman initially presenting with an S1 syndrome with lymphocytic pleocytosis in the CSF, leading to the misdiagnosis of meningoradiculitis. Repeated spinal MRI disclosed an epidural mass lesion, and histological examination of the tumour biopsy confirmed the diagnosis of primary spinal lymphoma of Burkitt's type. The authors emphasise that in every case with suspected meningoradiculitis including neuroborreliosis, meningeal lymphoma should be considered as an important differential diagnosis. In view of the occasionally difficult diagnosis of primary meningeal lymphoma we briefly present the clinicopathological report of a primary malignant lymphoma with almost selective involvement of the spinal roots: A woman aged 65 years without known systemic lymphoma presented with a painful focal right brachial plexus lesion and, 2 weeks later, developed progressive motor neuropathy with flaccic paraparesis of the lower limbs. Laboratory tests were negative except for her CSF showing increased protein content and 85 lymphocytes $/ \mathrm{mm}^{3}$, not expressing the B cell tumour marker CD 20. Nerve conduction studies suggested subchronic demyelinating neuropathy without motor conduction block. Cranial and cervical CT and MRI were negative. Sural nerve biopsy disclosd a demyelinating neuropathy with acute myelin loss some remyelination with incipient onion bulb formation, and wallerian degeneration, but without inflammatory or neoplastic infiltration. Muscle biospy showed neurogenic muscle atrophy. Despite high dose corticosteroid and intravenous immunoglobulin treatment, her condition deteriorated rapidly with develoment of flaccid paraplegia and paresis of the right arm. Repeated CSF analysis showed ncreasing protein content and pleocytosis up to 180 cells $/ \mathrm{mm}^{3}$, mainly transformed but with no neoplastic lymphocytes, again none being immunopositive for the $\mathrm{B}$ cell markers CD 19 and CD 20. Extensive search for extraneural malignancy including repeated cranial, spinal, and abdominal CT and MR scans were all negative. The patient died 6 months after the onset of neurological symptoms. Necropsy disclosed diffuse B cell lymphoma ${ }^{2}$ restricted to the cauda equina with massive infiltration of the anterior lum bal spinal nerve roots and dorsal ganglia, and very mild neoplastic infiltrates in the schiadic and femoral nerves associated with severe myelin loss. There was no involvement of the spinal cord, brain, meninges, or cranial nerves. The right brachial plexus showed severe demyelination without onion bulb formation and perivascular infiltrates exclusively formed by T lymphocytes. Search for systemic malignant lymphoma was negative except for an isolated small focal area of B cell lymphoma in the thyroid gland. ${ }^{3}$ As in the case reported by Wilkening et al, ${ }^{1}$ this finding widens the range of possibilities and differential diagnostic difficulties of selective involvement of the spinal roots (and peripheral nervous system) by primary malignant non-Hodgkin's lymphomas, often also referred to as "neurolymphomatosis"3-5 making an extensive diagnostic investigation necessary.

K A Jellinger

L Boltzmann Institute of Clinical Neurobiology, PKH/B-Building, Baumgartner Hoehe 1, A-1140

Vienna, Austria

W Grisold

L Boltzmann Institute of Neuro-Oncology, Kaiser Franz Josef-Spital, Kundratstraße 3, A-1 100

Vienna, Austria

Correspondence to: Dr K A Jellinger; kurt.jellinger@univie.ac.at

\section{References}

1 Wilkening A, Brack $M$, Brandis A, et al Unusual presentation of a primary spinal Burkitt's lymphoma. J Neurol Neurosurg Psychiatry 2001 :70:794-7.

2 The non-Hodgkin's lymphoma pathologic classification project. National Cancer Institute sponsored study of classification of non-Hodkin's lymphomas: summary and description of a working formulation for clinical usage. Cancer 1982;49:2112-35.

3 Grisold W, Jellinger K, Vass A, et al. Selective involvement of the peripheral nervous system in B-cell lymphomas (human neurolymphomatosis) [abstract]. J Neurol 1992;239(suppl 2):S12

4 Diaz-Arrastia R, Younger DS, Hair L, et al Neurolymphomatosis: a clinicopathologic syndrome re-emerges. Neurology 1992:42: 1 136-41.

5 van den Bent MJ, de Bruin HG, Bos GM, et al. Negative sural nerve biopsy in neurolymphomatosis. J Neurol

1999;246: $1159-63$.

\section{Authors' reply}

We greatly appreciate the interesting comments of Jellinger and Grisold who describe 
another unusual case of primary spinal lymphoma. In their case, by contrast with that presented by us the spinal roots were selectively involved without the formation of a mass lesion during the disease. Moreover, CSF studies in their case showed increased protein content and pleocytosis but no neoplastic cells, which had been found in our patient. Together, the case presented by us and the case of Jellinger and Grisold underlines the fact that the range of primary cerebral and especially primary spinal lymphoma is very wide with different clinical presentation, location of manifestation, prognosis, and probably of aetiology. More cliniconeuropathological studies on primary spinal lymphoma are necessary to better understand this disease and to develop therapeutic strategies for its different types.

A Wilkening, M Brack, $\mathrm{F}$ Heidenreich, $\mathbf{R}$ Dengler, K Weißenborn Department of Neurology, Medical School of Hannover, Carl-Neuberg Strasse 1, D-30625 Hannover, Germany

A Brandis Department of Neuropathology

Correspondence to: Dr A Wilkening; Wilkening.Anja@mh-hannover.de

\section{Orientation agnosia in pentagon copying}

Ala et $a l^{1}$ have recently claimed that patients with Alzheimer's disease (AD) were less likely to make errors on the pentagon copying component of the mini mental state examination (MMSE) than patients affected by Lewy body (LB) dementia. Using (standard and modified) MMSE scoring criteria they found normal copies in 16 of 27 patients with AD but in only two of 13 patients with LB. Although this difference was non-significant, ${ }^{1}$ they concluded that this feature might be useful in differential diagnosis. It is of some interest that the MMSE scoring criteria employed by Ala et al did not regard errors of rotation as abnormal. In the light of recently identified visuospatial deficits which affect the domain of orientation, it may well be that the criteria of Ala et al for a "normal" copy are inappropriate. This has implications for the claimed diagnostic power of their measure.

Patients with orientation agnosia are able to recognise objects, but have a selective visuospatial impairment, in that they have lost knowledge of object orientation. ${ }^{2}$ The most striking feature of this disorder is shown in the patients' drawing of objects, where they rotate their copy by 90 or 180 degrees relative to the original. This previously underinvestigated deficit is far from rare. In one study, seven of $63(11 \%)$ patients with stroke grossly rotated their copy of the MMSE. ${ }^{3}$ All had a right hemisphere lesion and showed some evidence of left visuospatial neglect. In a second study 16 of $240(7 \%)$ of a series unselected by pathology showed the same sign, but without clear lateralising features.

To better investigate this issue in degenerative disease, we reviewed the records of a consecutive series of 134 patients with AD. All had been given the MMSE, which includes the interlocking pentagon figure (aligned horizontally) as a copy task, and a battery of neuropsychological tests. Errors in copying the geometrical figure were systematically graded. In general agreement with the findings of Ala et al, 57 of 134 (43\%) drew unrecognisable figures, adding or omitting details.
Three showed signs of closing in, and seven ( $5 \%$ of the sample) made clear rotation errors, misplacing one or both pentagons by 90,120 , or 180 degrees relative to the original. These seven patients did not differ from the overall sample in terms of general severity of dementia, as measured by the MMSE total score, and by daily activity scales. Eight patients (6\%) presented with signs of visuospatial neglect, ${ }^{5}$ but patients with neglect and orientation agnosia did not overlap in this sample, suggesting that orientation agnosia should be considered an independent sign. Our data thus suggest that orientation agnosia is a significant visuospatial deficit in degenerative disease, which is not identified as such in the study of Ala et al by virtue of the criteria by which they define an abnormal performance.

In figure 2 of the paper of Ala et al, it is clear that some copies, scored as errorless, showed clear signs of orientation agnosia (AD cases 3 , $9,15,18,19$, and 24). If these errors are considered, the claimed difference between $\mathrm{AD}$ and $\mathrm{LB}$ dementia disappears, confirming the results of previous studies which showed no significant difference in drawing or constructional abilities between $\mathrm{AD}$ and $\mathrm{LB}$ dementia. ${ }^{6}$

S Della Sala Department of Psychology, King's College, University of Aberdeen, Aberdeen AB24 2UB, UK

O Turnbull

School of Psychology, University of Wales, Bangor, Wales, UK

N Beschin

M Perini

Department of Rehabilitation and Department of Neurology, Gallarate Hospital, Italy

Correspondence to: Professor S Della Sala; sergio@abdn.ac.uk

\section{References}

1 Ala TA, Hughes LF, Kyrouac GA, et al. Pentagon copying is more impaired in dementia with Lewy bodies than in Alzheimer's disease. J Neurol Neurosurg Psychiatry 2001;70:483-8.

2 Turnbull $\mathrm{OH}$, Beschin N, Della Sala S. Agnosia for object orientation: implications for theories of object recognition.

Neuropsychologia 1997;35:153-63.

3 Turnbull OH, Beschin N, Della Sala S.

Rotated drawing: a mini mental state examination performance with strong lateralising significance. J Neurol Neurosurg Psychiatry 1997;62:419-20.

4 Solms M, Turnbull OH, Kaplan-Solms K, et al. Rotated drawing: the range of performance, and anatomical correlates, in a series of 16 patients. Brain Cogn 1998:38:358-68.

5 Venneri A, Pentore S, Cotticelli B, et al. Unilateral spatial neglect in late stage of Alzheimer disease. Cortex 1998;34:743-52.

6 Heyman A, Fillenbaum GG, Gearing M,

et al. Comparison of Lewy body variant of Alzheimer's disease: Consortium to Establish a Registry for Alzheimer's Disease, Part XIX. Neurology 1999;52:1839-44.

\section{Authors' reply}

We were very pleased to see the interest that Della Sala and colleagues have shown in our study. ${ }^{1}$ Orientation agnosia is certainly a fascinating subject, and we applaud their research into its neuropsychology. We think, however, that their analysis of our our study has several weaknesses.

Firstly, because we had not included the original interlocking pentagon models with the copies in our figures, they incorrectly inferred that six of our patients with $\mathrm{AD}$ made rotational errors. As mentioned in our results, ${ }^{1}$ many different printed models of the interlocking pentagons were used in our study. To clarify for your readers exactly what the patients were shown to copy and how they performed, Figure 1 presents the models and the patients' copies for AD cases 3, 9, 15, 18, 19, and 24. To allow the study of rotational changes, the exact orientation to the bottom of the page is preserved for each model and for each copy. Although all have been reduced for publication, the relative sizes of the models and the copies are also preserved. By our inspection none of the questioned cases exhibits any significant rotational error. We also studied the copies of the remaining patients with $\mathrm{AD}$ and the patients with DLB in our study, and we could not form any conclusions about rotational errors with either group.

It should also be noted that we specifically used the original published criteria ${ }^{2}$ for grading the copies of the interlocking pentagons in the MMSE. These criteria state that "tremor and rotation are ignored" (table 1 of our article ${ }^{1}$.

The diagnoses of our patients were neuropathologically proved. It would be interesting to know if the diagnoses of the 134 patients with $\mathrm{AD}$ that Della Sala and colleagues mention were also neuropathologically proved. If their patients were diagnosed clinically, we

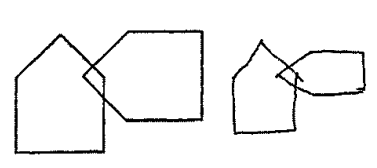

AD-3

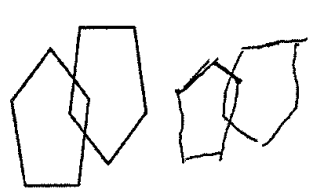

AD-18

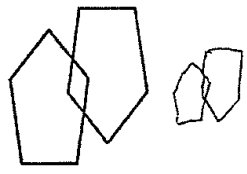

AD-9

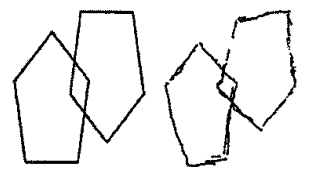

AD-19

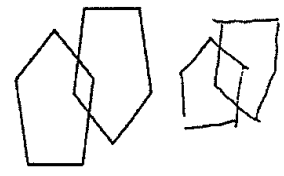

AD-15

Figure 1 Interlocking pentagon models and copies from the mini mental state examinations (MMSEs) of six patients with AD. For each pair, the model shown to the patient is on the left and the patient's copy is on the right. Each patient was included among the patients with AD reported in our original article, ${ }^{1}$ identified by the same code. Orientation to the bottom of the page and relative sizes within each pair are preserved. Each model-copy pair has been reduced in size for this figure. 
would not be surprised if some patients with DLB were included in the 134. Perhaps many of those who had the orientation agnosia were actually patients with DLB who did not exhibit the classic DLB clinical symptoms of fluctuation, visual hallucinations, and parkinsonism.

Also, the study ${ }^{3}$ that Della Sala and colleagues cite as evidence that there is "no significant difference in drawing or constructional abilities between AD and LB dementia" cannot be easily compared with ours because of the different severities of the patients involved. Based on MMSE scores, at least one half of the patients in that study were more severely impaired (median AD MMSE 10.5, DLB 9.0) than any of the patients in our study (minimum MMSE 13). That study does help confirm that constructional abilities worsen as the dementia worsens, for as we mentioned in our results, ${ }^{1}$ none of the additional cases of $\mathrm{AD}$ and DLB in our brain bank with initial MMSE scores less than 13 had acceptable pentagon copies.

Finally, albeit a minor detail at variance with Della Sala and colleagues, we did report that the DLB group copied the pentagons significantly less accurately than the AD group $(\mathrm{p}=0.002$ using the original criteria) .

T A Ala

Center for Alzheimer Disease and Related Disorders, Southern Ilinois University Medical School, P O Box 19643, Springfield, IL 62794-9643, USA

\section{References}

1 Ala TA, Hughes LF, Kyrouac GA, et al. Pentagon copying is more impaired in dementia with Lewy bodies than in Alzheimer disease. J Neurol Neurosurg Psychiatry 2001;70:483-8.

2 Folstein MF, Folstein SE, McHugh PR. "Mini-mental state". A practical method for grading the cognitive state of patients for the clinician. J Psychol Res 1975;12:189-98.

3 Heyman A, Fillenbaum GG, Gearing $M$, et al. Comparison of Lewy body variant of Alzheimer's disease with pure Alzheimer's disease: Consortium to Establish a Registry for Alzheimer's Disease, Part XIX. Neurology

1999;52:1839-44.

\section{BOOK REVIEWS}

\section{Advances in research on neurodegeneration. Volume 7}

By Yoshikuni Mizuno, Donald B Calne, Reinhard Horowski, Werner Poewe, Peter Riederer, and Moussa Youdim ( $\mathrm{Pp} 214$ US $\$ 95.00$ ). Published by Springer-Verlag, Wien, 2000. ISBN 3-211-83485-0

The book is part of a series based on the proceedings of the International Winter Conference on Neurodegeneration and Neuroinflammation. This particular volume arose from the seventh meeting, which was held in Karuizawa, Japan in January 1999.

The conference brought together scientists from a wide range of research areas, under the broad heading of neurodegeneration, and their breadth of expertise is reflected in the eclectic mix of studies presented here. The papers fall into four general categories, paralleling the scientific sessions of the meeting The first deals with progress in elucidating some of the genes and gene products involved in neurodegenerative processes. It includes an interesting paper on the cleavage of huntingtin by caspases and the possibility of using caspase inhibitors to stop the aggregation and hence neurotoxicity of the protein in Huntington's disease. This section also includes papers on inherited Parkinson's disease, both autosomal dominant and recessive mechanisms, with particular attention being paid to the role of the PARKIN gene in the juvenile onset form of the disease.

The second, and largest, group of papers address the very topical subject of cell death mechanisms in neurodegeneration. The areas covered range from the role of the antiapoptotic protein $\mathrm{Bcl}-2$ in spinal muscular atrophy, in vitro experiments looking at the effect of $\alpha$-synuclein mutations in neuronal cell cultures, to the possible role of polymorphisms in the myelin oligodendrocyte glycoprotein (MOG) gene in models of multiple sclerosis. Perhaps surprisingly, there was relatively little on the toxicity associated with amyloid precursor protein processing in Alzheimer's disease. However, that may not have been such a bad thing in this context, as a thorough discussion of this topic could easily require a separate volume in itself. Also included in this section (although it's not entirely clear why) is an interesting reevaluation of basal ganglia circuitry, by one of the editors, Professor Riederer, based on a review of clinical and experimental evidence.

The third section reflects the burgeoning interest in the role of cytokines in neurodegeneration and includes papers on the role of cytokines in Parkinson's disease and in oligodendrocyte death. Unfortunately, there is no discussion of their role in Alzheimer's disease where their involvement has long been suspected. There has subsequently been a major increase of interest in this area with the discovery of polymorphisms within the genes for interleukin 1 and their effects on disease prevalence and age of onset.

The final section is more speculative and deals with some possible future therapeutic interventions. These include the use of adenoassociated viral vectors for gene therapy in Parkinson's desease, neuroprotective therapies in multiple sclerosis, and the possible efficacy of transglutaminase inhibitors in the treatment of polyglutamine disorders.

Overall, I found this an interesting volume to dip in to but it suffers from the common problem of all meeting proceedings in that two and a half years down the line some of the findings are already beginning to look somewhat dated. It is perhaps worth noting that, according to the publisher's website volume 8 of this series, based on the meeting in Bavaria in February 2000, is already available.

Stephen Gentleman

\section{EMG waveforms: video companion to electromyography and neuromuscular disorders}

By David C Preston and Barbara E Shapiro (Pp 42 and PAL video, £55.00). Published by Butterworth-Heinemann, Woburn, 2000. ISBN 0-75-067278-1.

The practice of electromyography, despite many advances in signal analysis, quantification, and computer modelling, remains to an extent an art. The experienced electromyographer relies as much, if not more so, on his ears as well as his eyes to recognise patterns of muscle electrical activity.

Transferring this experience to trainees is a challenge, and this collection of video clips covering spontaneous activity (fibrillation, complex repetitive discharges, fasciculations, and various forms of myotonia and neuromyotonia) and voluntary EMG activity is a useful addition to our material. Each clip shows a standard EMG machine screen with an audio soundtrack and is introduced by a static video frame. Clips run for up to a minute and often display waveforms at different time resolutions. The making of measurements is also shown. It would have been preferable for a voiceover to have introduced each clip to highlight the points to be made. Reading the accompanying booklet works but has less impact. Some screen shots either show redundant information or small characters indicating, for example, amplitude, too small to read.

Nevertheless this is a valuable resource to introduce trainees to the range of electrical muscle activity which might be encountered in clinical practice.

Kerry Mills

\section{Neuropsychological evaluation of the older adult: a clinician's guidebook}

By Joanne Green (Pp 311, US\$69.95) Published by Academic Press, San Diego, 2000. ISBN 0122981901

This book gives comprehensive and thorough up to date coverage of this topic. It starts from the premise that neuropsychological evaluation of older people is a useful and productive endeavour, and then sets out to assist in making the process as efficient as possible, while being clearly respectful and humane. It guides the reader through every aspect of the process, from tasks which need to be addressed before the patient arrives, through the clinical interview and the formal testing, onto interpretation of test findings and report writing, rounding off with providing feedback and follow up. The practical aspects are supported by example letters, documents, forms, and reports, together with 11 useful case studies. In addition there are chapters reviewing in detail assessment procedures for memory and other cognitive functions, and four useful chapters examining the neuropsychological profiles of the most common (and a few rarer) conditions likely to be encountered in clinical practice, from Alzheimer's disease through to normal pressure hydrocephalus. The important topic of depression, its assessment, and its impact on neuropsychological performance, receives a chapter in its own right.

The author is to be congratulated on producing a book which travels well outside the North American context in which it was written; by largely concentrating on the Wechsler tests (and the most up to date versions of them) the detailed discussion of tests and their uses and properties seems familiar and accessible. The book is soundly based in current research and theory-for example, on Lewy body dementia and the vascular dementias-but at the same time conveys a strong sense of clinical experience and wisdom. The reader will not find here a defence of the contribution of neuropsychology to the management of cognitive disorders in older people, but a better account of the state of the art would be difficult to imagine.

Robert Woods

\section{Thrombolytic therapy for stroke}

Edited by Patrick D Lyden (Pp 398 US\$125.00). Published by Humana Press, New Jersey, 2001. ISBN 0-896-03746-0

The end of the 20th century saw a transition in the approach to acute stroke from therapeutic nihilism to enthusiasm. Stroke is now 
seen as an emergency, warranting urgent assessment, investigation, and treatment. The positive results of the major randomised trial of thrombolysis in acute stroke have played a major part in stimulating this new approach, which is the topic of this publication. This is very much a hands on book, with much practical advice, ranging from detailed advice abou the assessment and selection of patients for thrombolysis to how to set up and deliver an emergency service for stroke. The book ranges from a detailed description of the evidence from clinical trials to advice based on personal experience (for example, what questions to ask to determine the time of onset accurately).

The book starts with a review of the pharmacology and haematology of thrombolysis, the causes of ischaemic stroke, and a description of the ischaemic penumbra. The last is the holy grail of thrombolysis and refers to the concept that a portion of ischaemic brain can be salvaged before undergoing irreversible infarction if treatment can be started early. As becomes evident on reading further, the evidence from the thrombolysis trials suggests that ischaemic penumbra is rarely present for longer than 2 or 3 hours after the onset of stroke. The book then proceeds to an excellent account of the early trials of thrombolysis. These included only a few patients, but the results show the thinking behind some of the protocol requirements proposed for the safe use of thrombolysis. Streptokinase proved to have a very high rate of haemorrhage and was abandoned. By contrast, early studies of recombinant tissue plasminogen activator (rt-PA) were more encouraging. It was certainly a sur prise to read that the first attempt to trea stroke using thrombolysis in three patients was in 1958 and that there were another 30 case series before the larger randomised trials were started. The results of the randomised National Institute of Neurological Diseases and Stroke (NINDS) Trial are described in detail. The positive results of this trial led to the licensing of rt-PA for acute stroke in the United States. The two ECASS trials conducted in Europe, in which the primary analyses were neutral, are dismissed in a few paragraphs because they primarily randomised patients within 6 hours and included very few patients within 3 hours of onset. Given the detailed discussion of each of the trials, it is surprisingly that the book does not include a meta-analysis putting togethe the results of the different trials, but others have done so in reviews and confirmed that there seems to be benefit in patients treated under 3 hours, with an unproved trend to benefit in patients treated from 3 to 6 hours. Nevertheless, the uncertainty about the interpretation of the different results from the trials of rt-PA in North America and Europe is probably the main reason which has prevented rt-PA from receiving a licence for acute stroke in Europe.

Most of the studies have concentrated on intravenous thrombolysis. Intra-arteria thrombolysis has the advantage of being able to confirm and target the site of persisten arterial occlusion and might reduce the risk of systemic complications, but not necessarily cerebral haemorrhage. The evidence for arterial thrombolysis in stroke is reviewed in a separate chapter and strongly suggests that treatment in selected patients is beneficial up to 6 hours from onset. However, the requirements for an instantly available interven tional neuroradiology team limit its applicability to only a few centres.

It is essential to be able to recognise the early changes of cerebral infarction on CT if thrombolysis is to be used safely and an excellent chapter follows on the techniques and interpretation of CT in acute stroke. How ever, there is no mention of the role of MR or new imaging techniques such as diffusion weighted imaging. The book concludes withsome interesting case history vignettes, illustrated by the relevant CT, inviting the reader to decide whether or not to thrombolyse the patient. Most cases receive thrombolysis, sometimes outside the NINDS protocol recommended elsewhere in the book, but the cases illustrate the need for detailed and rapid assessment of every patient.

This book is clearly primarily directed at the North American market, where intravenous thrombolysis is a licensed treatment for stroke. For example, one chapter is entitled "How to run a code stroke". The term code stroke was a new term to me and was not explained, but it soon transpired that the chapter provided detailed information on how to organise emergency services and respond to the arrival of an acute stroke, so that treatment with rt-PA can be started within the vital 3 hours.

If there is a criticism of this book, it is that it lacks balance despite the best of efforts of the authors. The benefits of thrombolysis are taken as proved and dramatic, although the fact that some experts are less enthusiastic is acknowledged. Lou Caplan was invited to contribute a chapter arguing against thrombolysis for stroke, but it is clear that by the time he came to write the chapter he was thoroughly in favour of thrombolysis, although he does argue for proper investigation before selection of patients. The disadvantages of concentrating on thrombolysis for stroke receive scant attention. For example, there is no mention of the problem of diversion of resources away from other patients with stroke, or the importance of developing comprehensive stroke services before introducing thrombolysis. Nowhere could I find mention of the importance of the numbers needed to treat for benefit or harm (about eight and 16 respectively) or of the fact that only a small proportion of patients admitted to hospital with stroke are eligible for thrombolysis in the first place (4\% in the NINDS study), although there is a chapter with very detailed calculations on the cost effectiveness of rt-PA treatment in the American setting. The book would certainly have been more balanced if contributions had been included describing the European perspective-for instance, from the Cochrane collaboration or from one of the organisers of the International Stroke Trial 3, which plans to re-examine the risks and benefits of intravenous thrombolysis from 0 to 6 hours

My criticisms are minor and the book is highly recommended as a guide to current practice for those contemplating introducing thrombolysis into their clinical practice. This will require education of the community, doctors, and emergency services to regard stroke as a "brain attack" requiring urgent admission to a specialised unit. The introduction of thrombolysis has an important influence on the way stroke is perceived. Although only a small proportion of patients benefit directly from thrombolysis, many more benefit from the earlier assessment and care by a specialised team that is an essential component of providing the rapid response needed for thrombolysis. Intravenous thrombolysis is an important advance in stroke treatment, which deserves to be more widely implemented in centres with the appropriate facilities and experience.

Martin Brown

\section{Neurobiology of spinal cord injury}

Edited by Robert G Kalb and Stephen $M$ Strittmatter (Pp284, \$125.00). Published by The Humana Press, New Jersey, 1999. ISBN 0-896-03672-3

"The future for regenerative therapies of the spinal cord is clearly very bright" - thus ends this volume in Humana's Contemporary Neuroscience series, which looks at different aspects of spinal cord injury with special attention to possible strategies for repair.

Mechanisms of neuronal damage after injury, locomotor function in the transsected spinal cord, axon extension and elongation, as well as pharmacological and cell transplantation strategies to encourage axon regrowth and remyelination of remaining fibres, and current therapies for patients with spinal injury are all covered. Individual chapters were contributed by experts in the field, many of whom carried out seminal work on the topics they describe. This ensures that the text is on the whole informed, up to date, and written with authority, although sometimes maybe a little too focused on subjects close to the authors' hearts. Consistency in style, layout, and detail between the different chapters is somewhat lacking, which doesn't make this a particularly enjoyable read.

Much of the book concentrates on the study of spinal cord injury models and the insights gained about mechanisms of cell death and loss of function as well as the usefulness of repair strategies, with two of the 11 chapters dedicated to cell transplants. The text devotes very little attention to the clinical relevance of experimental findings, and is not really concerned with experience and insights gained from human spinal cord injury. None the less, current pharmacological therapies for patients and their possible mechanisms of action are discussed in the last chapter.

This would be a good introduction for those who are unfamiliar with mechanisms of neuronal injury and repair, and also provides a good update on recent developments in experimental strategies for spinal repair. Those with some experience in this subject will probably find that this volume does not provide sufficient detail in most areas to be an essential addition to their bookshelf.

Given that many of the authors have spent much of their careers on possible strategies for spinal cord repair, it is perhaps not surprising that this book exudes a general optimism in this regard. Certainly, progress has been made in several different areas, which may one day bring substantial benefit to patients, either alone or combined-details of each, and how they may compliment each other-are probably the main things that the reader will take away from this.

Rike Zietlow

\section{Cognitive neurorehabilitation}

Edited by Donald T Stuss, Gordon Winocur, and lan H Robertson (Pp 385, £64.95). Published by Cambridge University Press, Cambridge, 1999. ISBN 0521581028

As the French term "re-education" implies, learning is a key process in rehabilitation. Nothing might seem more challenging than to effect useful learning in people with cognitive impairments. Until recently, a cloud of pessimism hung over cognitive deficits, but the later sections of this useful book provide 
authoritative evidence that interventions can produce positive changes in people with impairments in memory, in attention, perhaps in executive function, and in language processing.

Is it rehabilitation? Most of the convincing effects described here are at impairment rather than disability level and therefore are not necessarily positive rehabilitation outcomes. But the book takes account of social factors, as well as problematic psychological categories such as motivation, which provide a pragmatic context within which future interventions might operate. It seems likely that more will be achieved in this new field.

Is it cognitive? One source of pessimism about therapy for cognitive impairment has been the dead hand of verbal learning, the "old paradigm" for cognitive science. It is hard, and often futile, to teach word lists to people with amnesia. A much more achievable goal is to alter their behaviour. A sort of neobehaviourism is creeping into cognitive science, inspired by artificial learning (neural) networks. The most relevant aspect of a chapter on the potential benefits of constraining an unaffected limb in hemiplegia is the account of "learned non-use" as a possible barrier to inducing movement in the plegic limb. Might the same mechanism lead a person with aphasia to use less speech, or someone with amnesia to become more restricted? Learned non-use can be described in terms of operant conditioning, as can other therapeutically fruitful concepts such as errorless learning. A good deal of cognitive rehabilitation is not really cognitive. The restitution of cognition (in its classical sense, as the handling of information in the conscious domain) still eludes us. But this book provides some signposts along the way.

Christopher D Ward

\section{Neuropathology and genetics of dementia: advances in experimental medicine and biology, volume 487}

Edited by Markus Tolnay and Alphonse Probst. (Pp 270, £63). Published by Kluwer Academic/Plenum Publishers, New York, 2001. ISBN 0306465582

This book represents the Proceedings of the XVIIIth International Winter Meeting of the Swiss Society of Neuropathology, held in St Moritz in March 2000. This is written as a series of short articles and longer reviews, rather than the usual unstructured cluster of synopses of experimental findings presented orally at such scientific gatherings. Thematically, the review articles focus on the role of abnormal aggregated or misfolded proteins in the brains of persons with diverse neurodegenerative diseases. Accordingly, there are chapters detailing the pathology and genetics of tauopathies, amyloidopathies and synucleinopathies, coupled with other chapters covering ubiquitin and polyglutamine disorders, inclusion body myositis and neuronal ceroid lipofuscinoses. The possible role of mitochondrial dysfunction in such disorders is discussed. Overall, the chapters are of a uniformly high standard and of sufficient breadth and depth to be useful and interesting to the specialist and casual reader alike. Rapid publication has ensured that most contributions are still well within their shelf life, although the camera ready format in which the volume has been prepared means the inclusion of occasional typographical and referencing errors, and a rather poor reproduction quality to the figures, particularly the half tones. The last is a particular shortcoming, given the weight of text devoted to neuropathological description. None the less, there is much useful information in this book, set out in a readable and up to date manner. It can be recommended particularly to junior researchers and physicians, who will find it a comprehensive and easily accessible reference. Lastly, it is noted in the acknowledgements that the editors were ably and fortunately assisted by a certain Michelle Pfeiffersurely not that one!

D M A Mann

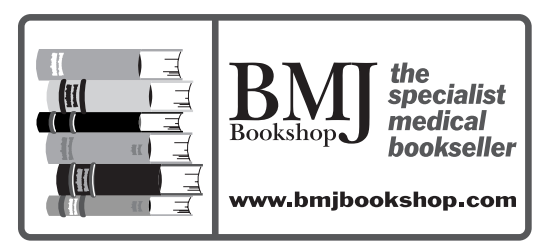




\section{Association of British Neurologists Autumn Meeting, Newcastle University meeting at Durham,} 12-14 September 2001

\section{A COMPARISON OF NEUROPSYCHOLOGICAL ASSESSMENT AND MRI IMAGING SCORES IN THE EARLY DIAGNOSIS OF ALZHEIMER'S DISEASE}

CJ Galton, R Swainson, R Vessy, BJ Sahakian, JR Hodges Addenbrookes Hospital, Cambridge, UK

The aim of this study is to address whether neuropsychological measures or imaging scores best predict which patients with questionable dementia progress to fulfil criteria for Alzheimer's disease (AD). MRI images of 50 subjects (18 with early AD, mean MMSE 21.4 , and 32 with memory complaints, mean MMSE 27.3) were examined using the temporal lobe rating scale. Subjects underwent a standard battery of neuropsychological tests at baseline, and were clinically followed up for a mean of 19 months. Patients who converted to $A D$ (converters $n=11$ ), relative to non-converters, were impaired at baseline assessment on general cognitive tests (Addenbrookes cognitive examination (ACE) and ADAS cog), measures of episodic memory, category fluency, and the graded naming test. The mean scores of the temporal lobe rating scale demonstrated that converters had more hippocampal and parahippocampal atrophy than non-converters. Discriminant analysis demonstrated that the best test for distinguishing the converters from the questionable group, was the ACE score. In combination, the medial temporal lobe rating and graded naming test were also contributory.

MRI imaging measures may be valuable if used in conjunction with neuropsychology, but progression to dementia is best predicted by performance on neuropsychological tests.

\section{EARLY COGNITIVE DECLINE IN CREUTZFELDT-JAKOB DISEASE OCCURRING IN RECIPIENTS OF PITUITARY-DERIVED HUMAN GROWTH HORMONE}

RJ Cordery, M Hall, L Cipolotti, L Davidson, P Adlard, MN Rossor National hospital for Neurology and Neurosurgery, London, UK

Most cases of Creutzfeldt-Jakob disease (CJD) in recipients of human cadaveric growth hormone, present with a cerebellar syndrome. Dementia is thought to occur late and as a minor feature of the illness. However, neuropsychology data published on these cases is largely qualitative and anecdotal. The first published case, of a 22 year old woman, does include neuropsychology performed 7 months after the onset of a cerebellar syndrome. This showed evidence of intellectual decline, with a verbal IQ of 80 . Subsequent reports hint at the possibility that cognitive problems may be present in the initial stages of the illness.

In order to assess early cognition, we report detailed assessment at referral (mean 4.5 months from the onset of symptoms, range 4-6 months) in five patients with histologically proved human growth hormone derived CJD. All presented with a cerebellar syndrome and only one had noticed mild memory problems. On formal testing however, four had demonstrable, mild intellectual decline, as measured on the WAIS-R. One case showed selective visual memory impairment and frontal executive dysfunction.

These findings suggest that, although not the presenting feature, mild cognitive decline may be evident in the early stages of CJD associated with human cadaveric growth hormone treatment.

\section{LATERALITY OF UNEXPLAINED MOTOR AND SENSORY SYMPTOMS: A SYSTEMATIC REVIEW 1965-2000}

J Stone, A Carson, R Goldbeck, S Lewis, B Thomas, CP Warlow, M Sharpe University of Edinburgh, UK

Background: Unilateral unexplained motor and sensory symptoms present commonly to neurologists. The literature has suggested that left sided symptoms are far more common than right.

Methods: A systematic review of the literature since 1965 to test this hypothesis. All studies describing one or more patients with unexplained, hysterical, psychogenic, or non-organic sensory or motor symptoms (excluding pain, visual, and auditory symptoms) were included.

Results: 124 studies with 1045 patients were analysed. The overall proportion of left sided weakness and sensory symptoms was $57 \%$
(Cl 53\%-60\%). This figure was accentuated in studies which specifically set out to study this question (64\% on the left). However, when laterality was reported incidentally there was no significant effect $(51 \%$ on the left). Unexplained movement disorders were left sided in 38\% (Cl 31-38\%). Female sex was related to left sided symptoms. Handedness and date of study did not influence laterality.

Interpretation: The data do not provide strong evidence for lateralisation of unexplained motor and sensory symptoms except for right sided movement disorder and possibly left sided symptoms in women. Retrospective studies specifically looking at lateralisation may have produced misleading positve results through bias. Consideration of the biology of these phenomena should not place undue weight on laterality.

\section{HAEMOGLOBIN CONCENTRATION AND STROKE RISK IN 13000 PATIENTS WITH ESTABLISHED CEREBROVASCULAR DISEASE}

SC Howard, DA Power, PM Rothwell on behalf of Cerebrovascular Cohort Studies Collaboration Radcliffe Infirmary, Oxford, UK

Small studies and case reports have suggested that polycythaemia may increase the risk of ischaemic stroke. There is physiological evidence to support this, but there have been no adequately powered cohort studies. We therefore pooled detailed individual patient data from cohort studies and clinical trials in which patients presenting with a TIA or non-disabling ischaemic stroke had haemoglobin measured at baseline and had been followed up.

Using data on 13009 patients with 60000 patient-years of follow up from eight independent studies, the relation between haemoglobin and risk of stroke was assessed by Cox regression adjusting for age, sex, other baseline factors associated with haemoglobin, and stratifying by study population.

Stroke risk had a " $U$ " shaped relation across eight predefined categories of haemoglobin concentration $(p=0.03)$. This was most marked for fatal ischaemic stroke $(p=0.001)$, haemorrhagic stroke $(p=0.02)$, and recurrent ischaemic stroke in patients presenting with a lacunar event $(p=0.005)$. The respective hazard ratios for haemoglobin above the normal range were $3.57(95 \% \mathrm{Cl}=1.87-6.84)$, $2.97(95 \% \mathrm{Cl}=1.07-8.20)$, and $1.49(95 \% \mathrm{Cl}=0.79-2.81)$.

Haemoglobin concentration is an independent predictor of ischaemic and haemorrhagic stroke in patients with established cerebrovascular disease. Correction of mild polycythaemia or anaemia may reduce the risk of stroke.

\section{A COMPARISON OF CT VERSUS DIFFUSION WEIGHTED IMAGING IN HYPERACUTE STROKE USING A SYSTEMATIC QUANTITATIVE SCORE (ASPECTS)}

PA Barber, MD Hill, AM Demchuk, JH Warwick Pexman, ME Hudon, A Tomanek, RR Frayne, AM Buchan for the ASPECTS Study Group Foothills Medical Centre, Calgary, Canada

Background: Tissue plasminogen activator (TPA) when administered $<3$ hours of symptom onset for acute ischaemic stroke produces improved functional and neurological outcome. Recent advances in neuroimaging with magnetic resonance (MR) diffusion weighted imaging (DWI) have raised hopes regarding the accurate identification of ischaemic tissue with improved safe administration of the treatment. CT is considered less sensitive and reliable than DWI for the detection of ischaemic change in hyperacute stroke. We hypothesised that CT is as good as DWI when the scans are read by experienced observers.

Methods: All patients had a CT scan (within 6 hours of stroke onset) prior to MRI ( $<7$ hours of onset). Non-contrast enhanced CT scans were performed on fourth generation scanners with $5 \mathrm{~mm}$ slice thickness parallel to the infraorbital meatal line, and appropriate window width (80 $\mathrm{HU})$ and centre level review settings (40 HU). MR imaging was performed on a 3Tesla machine and included DWI (single shot EPI TR=7000 $\mathrm{m}^{2} \mathrm{~s}^{-1}$, TE $=99 \mathrm{~ms}, \mathrm{~b}=1000 \mathrm{~mm} \mathrm{~s} \mathrm{~m}^{-1}, 195 \mathrm{~mm}$ slices $/ 3 \mathrm{~mm}$ gap). A quantitative scoring system (ASPECTS) was applied to CT and DWI at baseline and follow up ( $<36$ hours) by five 
independent observers (three stroke neurologists and two neuroradiologists) with only knowledge of the stroke side. The reliability of each modality was assessed for each observer with intraclass correlation coefficients. Bland Altman plots were used to measure the agreement between CT and DWI using ASPECTS.

Results: 80 patients $(65 \%$ male; mean age $68.4 \pm 13.2)$ had CT and DWI at baseline. The median NIHSS was 9. The mean onset to CT (136 minutes) compared with onset to MRI (240 minutes) $(p<0.001)$. The median baseline CT and DWI ASPECTS values were equal at 8 . Intraclass correlation coefficients confirmed excellent reliability between observers for CT $(0.81(95 \% \mathrm{Cl} 0.76-0.86))$ and DWI (0.78 (95\% Cl 0.72-0.84)) at baseline. CT ASPECTS predicted DWI ASPECTS (linear regression $p<0.001$ ) at baseline. The difference of means between DWI and CT at baseline was -0.3 ASPECTS, allowing for the mean difference in time for the scans.

Conclusions: CT appears comparable with DWI for assessing patients with hyperacute stroke when viewed by experienced observers using ASPECTS, and when sound CT imaging quality is achieved. The CT scan remains the imaging modality of choice in hyperacute stroke, while the challenge for MRI is to reliably identify salvageable brain tissue.

\section{HISTORY OF STROKE IN FIRST DEGREE RELATIVES OF PATIENTS WITH TIA AND ISCHAEMIC STROKE IN RELATION TO CLINICAL SUBTYPE AND INTERMEDIATE PHENOTYPES}

UGR Schulz, CP Warlow, PM Rothwell Radcliffe Infirmary, Oxford; Western General Hospital, Edinburgh, UK

Some rare mendelian stroke syndromes have been characterised, but the genetic epidemiology of the common forms of ischaemic stroke and TIA remains uncertain. Previous studies have not considered specific clinical and aetiological subtypes or assessed whether familial susceptibility is related to intermediate phenotypes.

We studied family history of stroke in 545 ischaemic strokes (Oxfordshire Community Stroke Project) and 469 TIAs (hospital based series). We related history of stroke in first degree relatives (FDRs) to baseline clinical characteristics, subtype of stroke, and risk of recurrence. TIAs.

Information on family history was available in 537 strokes and 467

Stroke had occurred in at least one FDR in $101 \quad 18.9 \%$, $95 \% \mathrm{Cl}=16-22)$ strokes and in $98(21 \%, 95 \% \mathrm{Cl}=17-25) \mathrm{TIAs}$. In both cohorts, a history of hypertension was strongly associated with family history $(p<0.01)$ and became more frequent with increasing numbers of affected FDRs $(p<0.01)$. However, there was no relation between family history and hyperlipidaemia, diabetes, stroke subtype, or risk of recurrence.

We found no evidence of a major independent genetic component in TIA and ischaemic stroke. However, the strong link between family history of stroke and hypertension suggests that genetic susceptibility to hypertension may explain a substantial proportion of the familial clustering of cerebral ischaemic events.

\section{A LATERALITY SHIFT OF BROCA'S AREA IN THE RECOVERY OF SPEECH PRODUCTION AFTER STROKE}

SC Blank, RJS Wise Hammersmith Hospital, London, UK

The production of both propositional speech $(\operatorname{PrSp})$ and nonpropositional speech (NPrSp) in normal subjects activates the left frontal operculum (Broca's area). We used PET to investigate speech production in five aphasic stroke patients with lesions that included the left frontal operculum.

All patients initially presented with severe non-fluent aphasia. Patients were scanned 16 months -10 years after stroke (median 4 years), when recovery of aphasia was deemed to be maximal. All subjects could count aloud fluently, but the extent of recovery of $\mathrm{PrSp}$ varied considerably, from occasional single word output to sentences.

Subjects were scanned under three experimental conditions; counting aloud (NPrSp), PrSp in response to autobiographical questions, and a non-speech state (XSp) when they attended to occasional environmental sounds. In normal subjects, PrSp and NPrSp contrasted with XSp revealed activation within Broca's area and the left anterior insula. In patients, activation was seen in the right frontal operculum. Thus, in patients with extensive damage to left premotor areas for articulation, homologous regions within the right hemisphere are recruited for the motor act of speech. This is direct evidence of plasticity within the neural systems engaged in speech production, and confirms Mohr's hypothesis about laterality shift of function in the frontal operculum.

\section{CLINICAL FEATURES AND DISEASE COURSE OF PSP IN THE UNITED KINGDOM}

U Nath, Y Ben-Shlomo, RG Thomson, N Wood, AJ Lees, DJ Burn Royal Victoria Infirmary, Newcastle upon Tyne, UK

Objective: To conduct a clinical survey of PSP and also to estimate its crude mortality rate in the United Kingdom.

Methods: 187 cases of PSP were identified in the context of a United Kingdom based prevalence study. Record-based confirmation of diagnosis according to the NINDS-SPSP criteria were performed in all cases and structural clinical assessment was also performed in a third of these cases. Information relating to subsequent death of patients was compared with national death certificate data collected all patients recorded as having PSP according to the ninth version of the International Classification of Diseases (ICD9)

Results: The median age of the clinical cohort was 72 years $(n=187)$. Non-specific visual problems such as diplopia and eyelid apraxia occurred in $39 \%$ and $17 \%$ of all patients but data on these items was not routinely recorded. Information from those patients undergoing standardised assessment $(n=49)$ revealed frequencies of $61 \%$ and $43 \%$ for these features respectively. Tremor was present in $21 \%$ of all cases and information from clinically examined cases suggested that this was usually postural. Early onset of diplopia and cases without tremor appeared to be associated with a shorter disease survival. PSP was mentioned in 57\% of death certificates of cases identified by record review.

Conclusion: PSP is frequently misdiagnosed. Visual symptoms are prominent and often disabling. Clinical factors such as early onset of diplopia are significantly associated with poorer survival. Mortality rate for PSP is significantly underestimated.

\section{PROGRESSION OF NIGROSTRIATAL DYSFUNCTION IN A PARKIN KINDRED: AN ${ }^{18}$ F-DOPA PET AND CLINICAL STUDY}

NL Khan, DJ Brooks, N Pavese, NW Wood, A Lees, P Piccini Institute of Neurology, Queen Square, London; Imperial College School of Medicine, Hammersmith Hospital, London; Royal Free Hospital and University College Medical School, London, UK

We have used ${ }^{18} \mathrm{~F}$-dopa PET to serially study a family with young onset parkinsonism with mutations in the parkin gene whose clinical picture is characterised by the presence of severe resting leg tremor. Four patients have been studied twice, 10 years apart, to assess disease progression. Additionally, we have studied five asymptomatic family members; four of whom carry a single parkin mutation and one individual with a normal genotype. Two of the carriers and the individual with the normal genotype had a repeat ${ }^{18} \mathrm{~F}$-dopa scan. In the parkin patients the rate of loss of ${ }^{18} \mathrm{~F}$-dopa uptake was similar in caudate and in putamen $(1.3 \%$ and $1.2 \%$ per annum respectively) indicating that disease progression is slower than that in idiopathic PD. The group of asymptomatic carriers also showed significant dopaminergic dysfunction in striatum bilaterally and in ventral midbrain and three of them developed subtle extrapyramidal signs. However in the two carriers scanned twice, ${ }^{18} \mathrm{~F}$-dopa dysfunction did not progress with time, at least over a 7 year period.

The slow rate of disease progression in parkin patients may explain the near normal longevity of some young onset PD patients. The development of mild signs of basal ganglia dysfunction in three carriers with abnormal striatal ${ }^{18} \mathrm{~F}$-dopa uptake suggests that these individuals are "manifesting heterozygotes" and may eventually develop parkin disease.

\section{UNILATERAL TRANSPLANTATION OF HUMAN PRIMARY FETAL TISSUE IN FOUR PATIENTS WITH HUNTINGTON'S DISEASE: NEST-UK SAFETY REPORT}

AE Rosser on behalf of the NEST-UK consortium Universities of Cardiff, Cambridge, Aberdeen, Belfast, London, and Manchester, UK

Huntington's disease (HD) is an inherited CAG repeat disorder typically manifesting in mid-life and leading to death within approximately 20 years. The principle pathology is focal degeneration of the major projection neurons of the striatum, making cell replacement by neural transplantation a potential treatment strategy. There is substantial experimental evidence that neural transplants can yield functional recovery in animal models of HD and several clinical trials have commenced. To date, only a few HD patients have received operations and the approach remains experimental. We report here the first phase of a Medical Research Council (MRC) supported clinical safety trial of neural transplantation in four patients with mild-moderate HD who have received unilateral grafts of human fetal striatal tissue. This is a multicentre UK-based trial affiliated to the organisation NECTAR 
(European Network for CNS transplantation and repair). At the time of writing all significant adverse events relate to the immunotherapy. There were no acute surgical events; no evidence of tissue contamination or overgrowth; and no evidence that the procedure has produced acceleration of the clinical course. Neural transplants would be expected to take at least 12 months to establish and to start to ameliorate function, thus the efficacy of these procedures will be reported at a later date.

\section{FAST MICROGRAPHIA AND PALLIDAL PATHOLOGY}

NP Quinn Institute of Neurology, Queen Square, London, UK

Micrographia is well recognised in Parkinson's disease (PD). Typically, the initial size and speed of the patient's handwriting is good, but rapidly becomes progressively smaller and slower, and may ultimately grind to a halt.

There exists another, quite distinct, form of micrographia which I call "fast micrographia", in which handwriting is microscopic from the outset, does not (indeed cannot) become progressively smaller, is accomplished at (astonishingly) normal speed, and does not fatigue. However, this micrographia can be so marked as to resemble a straight line, so that even the patients are often unable to read what they have written. In contrast with the micrographia of PD, many patients with fast micrographia have completely normal alternating finger movements.

Fast micrographia is most often observed in patients with progressive supranuclear palsy, who sometimes volunteer "microscopic" writing as their presenting complaint. However, it can also be seen in patients with acquired bilateral lesions of the globus pallidus. Illustrative videotapes will be shown.

Fast micrographia should be considered a clinical feature suggestive of underlying pallidal pathology.

\section{PROSPECTIVE STUDY OF DEVELOPMENT OF INFANTS BORN TO MOTHERS WITH EPILEPSY}

FH James, S Fairgrieve, SA Lynch, M Jackson University of Newcastle upon Tyne, Newcastle, UK

Objective: to establish if infants born to mothers with epilepsy are at increased risk of developmental delay.

Methods: Our prospective study of pregnant women with epilepsy identified 292 babies whose mothers gave consent to follow up with developmental assessment using Bayley's scales.

Results: We have examined 149 babies at 2-3 years to date: 59 were exposed to valproate, 61 to carbamazepine (50 in each group were on monotherapy). 31 children $(21 \%)$ have delayed development and $13(8.7 \%)$ have severe delay when the population prevalence of severe delay $=1.5 \%$ (OR 6.27, 95\% Cl 3.2-11.1). Developmental delay was associated with exposure to valproate IOR $4.42,95 \% \mathrm{Cl}$ 1.77-1 1.5). However, mothers of children with epilepsy had low educational achievement; only $47 \%$ achieved five GCSEs (grade A-G) compared with $85 \%$ of the population. Only $26 \%$ of mothers of delayed children obtained five GCSEs.

Conclusion: Children born to mothers with epilepsy in this study have increased risk of developmental delay at 2-3 years. The risk is increased with exposure to valproate and with maternal educational underachievement. Examination of the rest of the cohort followed by multivariate analysis will be undertaken in an attempt to identify the cause of developmental delay in these infants.

\section{QUANTITATIVE MRI ABNORMALITIES WITH HISTOPATHOLOGICAL CORRELATION IN REFRACTORY EPILEPSY: A TOOL TO PREDICT SURGICAL OUTCOME?}

TN Mitchell, SL Free, M Thorn, SM Sisodiya, SD Shorvon Institute of Neurology, Queen Square, London, UK

Focal cortical dysplasia (FCD) is the commonest neurodevelopmental lesion causing refractory epilepsy. 50\% of FCD patients have poor outcome after surgery, continuing seizures despite complete lesion resection, suggesting the presence, undetected on preoperative MRI, of additional epileptogenic abnormalities. Some forms of FCD may represent a forme fruste of tuberous sclerosis (TSC).

30 subjects with FCD, one with TSC, and 100 age matched controls had volumetric MRI scans. Quantitative analysis gave measures of regional distribution of grey and white matter and cortical thickness.

The TSC and $10(33 \%)$ FCD patients had additional extralesional abnormalities in areas that seemed normal on visual inspection of the MRI. The patient with TSC subsequently died. On postmortem, distinct pathological abnormalities (heterotopic grey matter, dysplastic giant neurons) were demonstrated in areas shown only by quantitative MRI analysis to be abnormal. The proportion with additional quantitative abnormalities, which could be additional subtle malformation, in our FCD group is comparable with that with a poor outcome after focal FCD resection.

Our technique could have potential application as a prognostic presurgical screening tool to prevent patients unlikely to achieve seizure freedom from being needlessly exposed to the risks of surgery.

\section{A PROFILE OF EPILEPSY IN A UK POPULATION}

NF Moran, K Poole, G Bell, J Solomon, S Kendall, M McCarthy, D McCormick, L Nashef, J Sander, SD Shorvon Institute of Neurology, Queen Square, London, UK

Objective: To describe the characteristics of epilepsy in a representative UK sample, including demographic features; onset age; duration; severity; antiepileptic drug (AED) use and the impact of epilepsy on life.

Methods: A large, geographically comprehensive survey using a postal questionnaire distributed by general practitioners to 3455 unselected patients receiving AEDs for epilepsy, in all UK regions.

Results: There were 1652 replies. The mean age was 44 years; $47 \%$ males. The mean onset age, 25 years, and the mean duration, 20 years, were comparable with epidemiological studies. In the preceding year, $52 \%$ of patients had no seizures; $8 \%$ a single seizure, $17 \% 2-9$ seizures, and $23 \%>10$ seizures; $64 \%$ had mild epilepsy and $32 \%$ severe. There was a marked and significant decrement of seizure frequency with increasing age. The most common AEDs were carbamazepine $(37 \%)$, valproate $(36 \%)$, phenytoin $(29 \%)$. Monotherapy was used in $68 \%$ of patients. Patients taking multiple AEDs reported significantly higher adverse effects. The major impacts on life were work and school difficulties, driving, and psychological, with variation according to age and severity.

Conclusions: Seizures remain uncontrolled in half of people with epilepsy in the UK with significant psychosocial impact. Our findings help address the deficiency of information on the characteristics of epilepsy in the elderly, in whom the condition is an increasing health problem.

\section{THE MANAGEMENT OF ADULTS WITH EPILEPSY}

D Smith, I Crook, IR Williams on behalf of the North West Clinical Neuroscience Partnership Project Walton Centre for Neurology and Neurosurgery, Liverpool, UK

Suspected and established epilepsy are common reasons for referral to hospital clinics. Optimal management, ideally, involves cooperation between an epilepsy clinic and primary care. Because these facilities are not widely available these patients can be exposed to misdiagnosis, incomplete diagnosis and suboptimal therapy and inadequate counselling. There is a need for expansion or reorganisation of services and concise guidelines could facilitate the care of these patients.

The North West Clinical Neuroscience Partnership project has developed a framework for service provision for adults with possible or definite epilepsy. A multidisciplinary team made contributions, based on reviews of available evidence, to the guidelines document. This document contains recommendations on (a) a structure for service provision, (b) diagnosis of epilepsy, and (c) the management and follow up of newly diagnosed and refractory epilepsy and includes specific advice regarding choice of treatment and the management of epilepsy in women, people with learning disability and the elderly.

This framework could facilitate best practice/shared care in either established or evolving services. Specific aspects of care are easily amenable to prospective audit. Adherence to guidelines is a transparent method of demonstrating the accountability of a service assuring commissioners/patients that a system capable of delivering quality care is in existence.

\section{CLINICAL FEATURES OF PARANEOPLASTIC NEUROLOGICAL DISORDERS: A RETROSPECTIVE STUDY}

JH Rees, I Sutton, R Weil, M Barnett National Hospital for Neurology and Neurosurgery, London, UK

There is a paucity of information about paraneoplastic neurological disorders (PND), a heterogeneous group of immune mediated conditions associated with a variety of different tumours. We report the preliminary results of a retrospective observational study investigating the clinical features of 57 patients with PND, ascertained principally via the British Neurological Surveillance Unit. The average (SD) age of onset was 63 (11) years and there was a female preponderance 
(3:1). The most often reported syndromes were sensory neuro(no)pathy $(33 \%)$ and cerebellar degeneration, either pure $(21 \%)$ or in association with brainstem/limbic encephalitis/neuropathy (23\%). SCLC was the most frequent underlying tumour type $(28 \%)$, followed by breast $(16 \%)$, ovarian $(9 \%)$ and other lung $(7 \%)$ carcinoma. No tumour was identified in $19 \%$. Forty six per cent of patients received immunomodulatory therapy and $67 \%$ of patients had their tumour treated. Only a minority of patients responded to these treatments and often only briefly. Progressive deterioration was the rule and $40 \%$ of patients with adequate follow up were dead at 12 months. We conclude that the diagnosis of PND leads to the detection of a tumour in $80 \%$ of cases but treatments are poor. More research is needed to improve the prognosis of these difficult conditions.

\section{AUDIT OF 2 WEEK URGENT REFERRALS FOR CNS/BRAIN TUMOURS}

AU Wills, DSNA Pengiran Tengah Queens Medical Centre, Nottingham; Derbyshire Royal Infirmary, Derby, UK

Objective: To audit patients referred from general practitioners (GPs) via the 2 week wait system and contrast with neurological cancer patients identified independently of this system.

Methods: GP referral letters were reviewed and compared with Department of Health (DoH) guidelines. Patient case notes were examined to determine the actual neurological diagnosis. A list of patients with cancer that were not identified by the system was compiled separately.

Results: Forty five patients were referred during a 9 month period, with 41 sets of case notes available. 13 referrals did not follow the DoH guidelines. Only four patients actually had CNS tumours (two astrocytomas, two cerebral metastases). The remainder were diagnosed with chronic daily headache (10), epilepsy (five), migraine (three), ?demyelination (two), essential tremor (two), other (16). At least 69 neurological cancers were identified during this period who were not referred via the 2 week system.

Conclusion: CNS cancers present in a diverse manner. Eliciting a neurological history and interpreting neurological signs can be challenging and many GPs have little neurological training. These guidelines, even when followed stringently, do not seem to increase diagnostic precision. Inappropriate referrals have extended already lengthy waiting times for neurology outpatient clinics and may have inadvertently delayed other patients with urgent neurological problems being seen. We suggest that the use of these guidelines in neurological practice is re-considered at the earliest opportunity. Perhaps a system of closer communication between primary and secondary care would be a more effective use of time and resources.

\section{IRREVERSIBLE DAMAGE TO THE SPINAL CORD AFTER SPINAL ANAESTHESIA}

K Hamandi, J Mottershead, T Lewis, IEC Ormerod, IT Ferguson Frenchay Hospital, Bristol, UK

Neurological features are a recognised rare complication of spinal and epidural anaesthesia. A number of mechanisms of injury to the spinal cord have been suggested. We report five cases of damage to the distal spinal cord after spinal anaesthesia. All patients were female undergoing orthopaedic (three patients) or obstetric (two patients) surgery. They had significant unilateral leg weakness and sensory disturbance after anaesthesia, which persisted at 2 year follow up. Three patients experienced pain on injection, the other two were under general anaesthetic at the time of spinal injection. Two patients were overweight and two had significant lumbar lordosis. MRI of the lumbosacral spine showed similar findings, in all cases, of high signal within the conus medullaris consistent with the clinical findings. Direct trauma was the most likely mechanism of injury. This is the first series to report detailed neuroimaging and long term follow up in all cases which shows that permanent neurological deficit has occurred. The use of spinal and epidural anaesthesia is becoming more widespread. Neurologists are likely to be involved in the management of complications and should be aware of this rare but serious complication.

\section{A DOUBLE BIND PLACEBO CONTROLLED STUDY OF INTRANASAL SUMATRIPTAN IN ACUTE CLUSTER HEADACHE}

PJ Goadsby, JA van Vliet, A Bahra, V Martin, SK Aurora, NT Mathew, MD Ferrari Leiden University Medical Centre, Leiden, The Netherlands; Cincinnati Headache Center, Cincinnati, USA; Headache Center, Seattle, USA; Houston Headache Clinic, Houston, USA; Institute of Neurology, Queen Square, London, UK

Cluster headache attacks reach a peak of pain intensity quickly, and are short lasting; therefore acute treatments must be rapidly acting.
This study sought to determine if intranasal sumatriptan is an effective treatment of acute cluster headache.

Patients with episodic and chronic cluster headache, by International Headache Society criteria, were recruited and after explanation and obtaining informed consent, were randomised to a double blind placebo controlled two period crossover study. Patients were instructed to treat two acute cluster headaches of at least moderate pain severity, with at least a 12 hour break, using intranasal sumatriptan $20 \mathrm{mg}$ or matching placebo. Patients recorded the time of onset of the attack, time of treatment, headache severity on a five point scale (0-nil, 1-mild, 2-moderate, 3-severe, 4-very severe) at 5, 10, 15, 20 and 30 minutes after treatment. The primary end point was the headache response defined as: very severe, severe or moderate pain becomes mild or nil, at 30 minutes. Secondary measures included pain free rates, treatment of associated symptoms and adverse events. The primary end point was analysed using a multilevel analysis approach with MLwiN (www.ioe.ac.uk/multilevel) with $p<0.05$ as the level of significance for testing.

In total, 118 patients were recruited; 97 males and 21 females. Of these 86 provided efficacy data on attack one and 80 on attack two. Twenty five provided no efficacy data because their bout ended, seven were lost to follow up. Six cycled out of a cluster period between attack one and attack two. Modelling the treatment outcome as a binomial where response was determined by treatment, using the patient as the level 2 variable, and considering period effect, sex, site and cluster headache type as other variables of interest, the effect of intranasal sumatriptan $20 \mathrm{mg}$ was superior to placebo at 30 minutes on the headache response end point $(p=0.01)$. For the first attack the placebo response rate was $11 / 37(30 \%)$ and the sumatriptan $20 \mathrm{mg}$ rate $29 / 50(58 \%)$; for the second attack the rates were 15/45 (33\%) and $18 / 36(50 \%)$, respectively. There were no serious adverse events.

It can be concluded that intranasal sumatriptan $20 \mathrm{mg}$ is effective in the acute treatment of cluster headache when compared with placebo. For cluster headache patients these data add a further evidence based treatment to the management portfolio of this disabling primary headache.

\section{EFFECT OF THE ADENOSINE (A1) RECEPTOR AGONIST GR79236 ON TRIGEMINAL NOCICEPTION WITH BLINK REFLEX RECORDINGS IN HEALTHY HUMAN SUBJECTS}

NJ Giffin, F Kowacs, V Libri, P Williams, PJ Goadsby, H Kaube Institute of Neurology, Queen Square, London, UK

There are no good migraine models in humans, but development of a low current intensity trigeminally mediated blink reflex (BR) has allowed the study of nociceptive transmission in the trigeminal nucleus caudalis. Adenosine is antinociceptive in humans and animals. GR79236 is a highly potent and selective adenosine Al receptor agonist. We investigated the effect of GR79236 on the nociceptive BR using 12 female healthy volunteers randomised in a placebo controlled double blind crossover study. BRs were elicited by supraorbital nerve stimulation with standard or nociception-specific electrodes at random intervals of 12-18s. BR responses were recorded bilaterally from infraorbital muscles. Each measurement was based on 25 sweeps. Areas under the curve (AUCs) of the R2 component of the BR were calculated from the rectified EMG, before and 30 minutes after drug (GR79236 (10 mg/kg)) or placebo. Primary end point: median $A U C$ at 30 minutes, modelled using analysis of covariance.

There was a non-significant reduction of the ipsilateral nociceptive R2 after GR79236 vs placebo of $17 \%$. However, there was a significant reduction of the contralateral nociceptive R2 $(p<0.05)$ of $20 \%$. There were no significant adverse events.

The results suggest that GR79236 may inhibit trigeminal nociceptive pathways. The nociception specific electrode directly depolarises cutaneous fibres and GR79236 may therefore act at second order trigeminal neurons in the nucleus caudalis, or more rostrally, crossing the blood-brain barrier. This suggests that it may be effective in primary headache disorders.

\section{A STUDY OF THE ACQUIRED NEUROMYOTONIA PHENOTYPE OF PERIPHERAL NERVE HYPEREXCITABILITY}

P Maddison, I Hart, K Mills, A Vincent, J Newsom-Davis Radcliffe Infirmary, Oxford; University of Liverpool, Liverpool; The Walton Centre for Neurology and Neurosurgery, Liverpool; King's College Hospital, London, UK

Acquired neuromyotonia (NMT) and cramp fasciculation syndrome (CFS) represent the severe and mild manifestations of generalised peripheral nerve hyperexcitability $(\mathrm{PNH})$ respectively. Anti-voltage gated potassium channel (VGKC) antibodies have been implicated in autoimmune PNH disease pathogenesis. We studied 44 consecutive 
patients with NMT seen in two centres to determine the significant clinical, immunological, and electrophysiological features of this phenotype.

The mean age at symptomatic onset was 47 years (range 9-74): over half $(64 \%)$ were male. Associated autoimmune diseases and other autoantibodies were found in $22(50 \%)$ : an immunoprecipitation assay detected raised titres of anti-VGKC antibodies in 16 out of 40 $(40 \%)$ patients.

All patients had at least two of the three principal symptoms of muscle twitching, cramps, and muscle stiffness. Other often observed features were increased sweating in $25(57 \%)$ muscle weakness in 16 $(34 \%)$, and pseudomyotonia in $16(34 \%)$.

Spontaneous, abnormal activity detected electromyographically comprised characteristic doublet, triplet, and multiplet motor unit (or partial motor unit) discharges, in addition to fasciculations and fibrillations. The most common abnormality on EMG, in all but three patients, were doublet discharges, with intraburst frequencies often exceeding $100 \mathrm{~Hz}$ (mean $168 \mathrm{~Hz}$, range $40-280 \mathrm{~Hz}$ ). Multiplets (runs of $>3$ discharges) were found in the remainder.

NMT is a phenotype of autoimmune PNH that seems to be distinct from CFS only in terms of the severity of symptoms, signs and EMG features observed, with both sharing immunological associations.

\section{HETEROPLASMIC MITOCHONDRIAL DNA MUTATIONS: DETERMINING THE MECHANISM OF INHERITANCE}

PF Chinnery, DC Samuels, DT Brown, DM Turnbull University of Newcastle upon Tyne, UK

Mitochondrial DNA (mtDNA) mutations cause progressive disabling neurological disease in at least 1 in 8000 of the UK population. Many mtDNA mutations are heteroplasmic, with a mixture of mutant and wild type mtDNA present within the same individual. A higher percentage level of mutant m+DNA (mutation load) is associated with more severe disease. Females harbouring heteroplasmic mtDNA point mutations transmit a variable amount of mutant mtDNA to their offspring, making it extremely difficult to predict the outcome of pregnancy. In mice, the variation in mutation load between offspring is determined by random genetic drift and a restriction in the number of mtDNA molecules early in embryogensis (the mitochondrial "genetic bottleneck"). It remains to be established whether the same mechanism operates in humans. We studied the mechanism of inheritance of the most common transmitted heteroplasmic mtDNA defect: the A3243G ("MELAS") mutation. By measuring mutation load in 82 individual human primary oocytes we show that random drift is the principal mechanism behind the transmission of heteroplasmy in human pedigrees with mtDNA disease. We also provide the first direct evidence of a mitochondrial genetic bottleneck in humans, ennabling the first direct measurement of bottleneck size. These findings will have important implications for genetic counselling.

\section{RESULTS OF A UNITED KINGDOM-WIDE CLINICAL AND MOLECULAR GENETIC STUDY OF MYOTONIA CONGENITA}

NP Davies, LH Eunson, MG Hanna Institute of Neurology, Queen Square, London, UK

Myotonia congenita is the commonest skeletal muscle channelopathy and is caused by mutations in the skeletal muscle chloride channel gene (CLCN1) on chromosome $7 q 35$. The aims of the study were to analyse the clinical and genetic features of myotonia congenita in the UK, to look at genotype-phenotype correlation in this group, and to develop a DNA based diagnostic service

In collaboration with the British Neurological Surveillance Unit we identified over 100 families with inherited myotonia. We initially performed SSCP analysis of all 23 exons of the CLCN1 gene in index cases from 42 families. In order to check that no mutations had been missed with this technique we subsequently analysed all the exons by direct DNA sequencing.

Using SSCP analysis mutations were identified in $50 \%$ of cases. This figure rose to $65 \%$ with DNA sequencing. In this mutation positive group the age at onset varied from $1-16$ years (mean 5.6 ), muscle pain was reported in $20 \%$ and $50 \%$ reported exacerbation of muscle stiffness in a cold environment. Twelve novel mutations have been identified. In particular the finding of a severe myotonic phenotype in association with compound heterozygosity and a mild phenotype with a new homozygous nonsense mutation (C242X) will be highlighted.

DNA based diagnosis is a realistic option in myotonia congenita. Although the mutations identified are spread across the gene it seems that exon 8 is a hot spot in this population. Genetic counselling in this disorder is complex and should include consideration of both genetic and functional expression data if available.

\section{A CYSTEINE RESIDUE CLOSE TO THE C-TERMINUS OF THE E SUBUNIT IS ESSENTIAL FOR SURFACE ACETYLCHOLINE RECEPTOR (ACHR) EXPRESSION: IMPLICATIONS FOR ACHR DEFICIENCY SYNDROME}

J Ealing, S Brownlow, M Al-Murani, M Brydson, A Vincent, D Vaux, D Beeson John Radcliffe Hospital, Oxford; University of Oxford, Oxford, UK

AChR deficiency is a recessively inherited form of congenital myasthenia in which adult AChR $\left(\alpha_{2} \beta \delta \epsilon\right)$ are severely reduced or absent at the neuromuscular junction. We have identified two new mutations at the extreme $C$ terminus of the $\epsilon$ subunit in AChR deficiency patients and have investigated the mechanism by which such distal mutations may affect expression.

Naturally occurring and artificial mutations were introduced into the $\epsilon$ subunit cDNA by in vitro mutagenesis. Wild type or mutant $\epsilon$ subunit cDNAs were transfected into HEK 293 cells with cDNAs for the $\alpha, \beta$, and $\delta$ AChR subunits. Surface expression of AChR was quantified by radioimmunoprecipitation of ${ }^{125} \mathrm{l}-\alpha$-bungarotoxin labelled receptor using an $\epsilon$ specific antisera. To determine the subcellular location of $\epsilon$ subunits, fluorescently labelled $\epsilon$ subunits were visualised using confocal microscopy. Lack of a cysteine residue (Cys 470), four amino acids from the $C$ terminus of the $\epsilon$ subunit, effectively abolished surface expression of the intact receptor in HEK 293 cells. Preliminary confocal data suggests that the mutant $\epsilon$ subunits accumulate in the endoplasmic reticulum.

These novel results reveal a previously unidentified signal sequence involved in the assembly and/or transport of AChR. Additionally, these results provide a mechanism by which frameshift or non-sense mutations in the $C$ terminus region of the e subunit lead to $A C h R$ deficiency.

\section{SERUM AUTOANTIBODIES TO OLIGODENDROCYTE AND NEURONAL CELL SURFACE ANTIGENS IN MULTIPLE SCLEROSIS PATIENTS}

O Lily, J Palace, A Vincent Radcliffe Infirmary, Oxford, UK

Recent evidence suggests that autoantibodies may be important in the pathogenesis of multiple sclerosis (MS), but the antigens to which they bind remain unknown. We incubated cultured human CNS cell lines with sera from MS patients and other inflammatory neurological disease controls (OIND). Surface binding was measured using flow cytometry and considered positive if greater than the mean +2 standard deviations of 12 healthy controls.

Antibody binding to two control cell lines was similar using MS or non-MS sera. By contrast, 70\% (16/23) of secondary progressive (SP) MS sera demonstrated positive binding to SK-N-SH neuroblastoma cells compared with $29 \%$ (10/35) of relapsing remitting (RR) patients $(p<0.001)$ and $0 / 16$ OIND controls. Also, 40\% of MS sera showed positive binding to $\mathrm{HOG}$ oligodendroglioma cells with no significant difference between RR and SP patients. Differentiation of the oligodendroglial cells resulted in the loss of positive antibody binding seen using MS sera, suggesting that the relevant antigens are present on oligodendrocyte precursor cells rather than mature oligodendrocytes. Sera with antineuronal antibodies generally also had antioligodendroglial antibodies, although antibodies against one cell line could not be absorbed by the other. These results support reports of heterogeneity in MS pathology, with autoantibody mediated processes important in a subgroup of patients.

\section{NON-INVASIVE VENTILATION IN MOTOR NEURON DISEASE: CURRENT UK PRACTICE}

SC Bourke, PJ Shaw, T Williams, GJ Gibson University of Newcastle; University of Sheffield; Royal Victoria Infirmary, Newcastle, UK

Background: The clinical application of non-invasive ventilation (NIV) in motor neuron disease (MND) has not been reported in the UK.

Methods: A questionnaire assessing clinical practice over the previous 12 months was sent to all UK consultant neurologists, with a second mailing to non-responders after 6 weeks.

Results: The response rate was $75.9 \%$. 1719 new patients were diagnosed, and 2280 patients were under review. 234 patients were referred for NIV and 126 were currently receiving NIV. Most responders $(172 / 265)$ did not refer any patients for NIV, however three neurologists made $30 \%$ of all referrals. The referral rate correlated with the number of new patients seen $(r=0.40, p<0.00001)$.

Referral for NIV was based mainly on symptoms rather than physiological impairment. $73 \%$ of responders measured vital capacity, and less than $50 \%$ checked blood gases or serum bicarbonate. Referral was influenced by level of dependence, rate of progression, and bulbar involvement. 
Conclusion: In the UK only a small proportion of MND patients are referred for NIV, with marked heterogeneity of practice. This may reflect concerns that NIV may prolong suffering, and emphasises the need for a controlled trial assessing its impact on quality of life.

\section{AN MRI STUDY OF COMPLICATED EARLY CHILDHOOD CONVULSION}

RA Grünewald, T Farrow, CDC Rittey, P Vaughan, J Mundy Royal Hallamshire Hospital; Ryegate Children's Centre, Sheffield, UK

Objectives: About half of adults with hippocampal sclerosis have a history of complicated early childhood convulsion (cECC). The objective of the study was to assess the extent of structural brain abnormality present soon after a first CECC.

Methods: Children under the age of 5 years underwent brain MRI within 14 days of their first complicated febrile or non-febrile ECC. None had previously experienced an epileptic seizure. Hippocampal volumes and T2 relaxation times were measured. The control group consisted of children undergoing brain MRI for reasons other than epilepsy.

Results: Nine of the 17 experimental subjects had significant hippocampal volume asymmetry $(3$ standard deviations from the mean of the control group), although in only three of these was the asymmetry apparent on visual inspection of the MRI. Three experimental subjects had extrahippocampal neuropathology. None of the 10 control subjects had significant hippocampal volume asymmetry $(p<0.001)$. T2 relaxometry did not suggest that oedema contributed to the asymmetry.

Conclusions: There is a high prevalence of structural brain abnormalities and hippocampal asymmetry in children within 2 weeks of the first complicated early childhood convulsion, unrelated to oedema. This suggests that in some cases the complicated EEC is the result of pre-existing brain abnormalities.

\section{A DOUBLE BLIND SINGLE DOSE CROSS OVER STUDY OF THE EFFECTS OF PRAMIPEXOLE, PERGOLIDE, AND PLACEBO ON TREMOR AND UPDRS (III) IN PARKINSON'S DISEASE}

P Navan, LJ Findley, R Pearce, J Jeffs, PG Bain Imperial College School of Medicine, London; Havering Hospital NHS Trust, Essex; Hammersmith Hospital, London UK

Design: 10 tremulous PD patients were pretreated with domperidone and taken off anti-parkinsonian medication. Each patient received single doses of $0.5 \mathrm{mg}$ pramipexole, $0.5 \mathrm{mg}$ pergolide, and placebo, but in random order. Rest tremor and UPDRS (part III) were measured at baseline and 30 minute intervals for 4 hours. The results were examined using ANOVA by area under the curve (AUC) and peak effect (Bonferroni corrected; significance: $5 \%$ level)

Results: (1) AUC analysis showed a significant difference between the three treatments on rest tremor $(p=0.045)$ (pramipexole differed from placebo at $10 \%$ level) but not UPDRS (III). (2) Peak effect analysis demonstrated a difference in treatment effects on UPDRS (III) $(p=0.04)$ (pergolide better than placebo, $p=0.048)$ and rest tremor $(p=0.005)$ (no significant difference between active drugs, both significantly better than placebo). Pergolide caused a significantly greater incidence of nausea $(p=0.005)$ and vomiting $(p=0.014)$ than pramipexole. No significant differences between the 3 treatments on incidence of drowsiness or sleep (McNemar's test).

Conclusion: $0.5 \mathrm{mg}$ pramipexole or pergolide can improve UPDRS (III) and rest tremor in PD. Pergolide had the strongest effect on peak UPDRS (III), whereas pramipexole had the greatest effect on rest tremor (AUC). Pramipexole is significantly better tolerated than pergolide.

\section{PALLIDAL STIMULATION FOR UNUSUAL MOVEMENT DISORDERS: 1 YEAR OUTCOME OF FOUR PATIENTS}

SG Parkin, RP Gregory, TZ Aziz The Radcliffe Infirmary, Oxford, UK

Pallidal stimulation has become an established mode of treatment in Parkinson's disease when medical therapy has failed. There is historical evidence to suggest that surgery to the basal ganglia can benefit a wide range of movement disorders. The relative safety of deep brain stimulation has allowed us to re-explore its benefit to other, less common conditions. We report the outcome of pallidal stimulation in a consecutive series of patients with idiopathic generalised dystonia, familial myoclonic dystonia, idiopathic progressive chorea, and tardive dyskinesia with captocormia. All had failed an exhaustive trial of medical therapy. Highly significant benefits were seen in various movement disorder rating scales and quality of life measures. These were maintained at 1 year. The obvious clinical benefits, rarity and heterogeneity of conditions of this type, precludes the use of randomised clinical trials. Detailed follow up of individual patients is important to provide evidence for the application of pallidal DBS in such conditions.

\section{CRITERIA FOR INITIATING NON-INVASIVE VENTILATION IN MOTOR NEURON DISEASE}

SC Bourke, PJ Shaw, R Bullock, GJ Gibson University of Newcastle, Newcastle; University of Sheffield, Sheffield; Newcastle General Hospital, Newcastle, UK

Objective: We studied the criteria which best predict benefit from quality of life (QOL) and compliance with non-invasive ventilation (NIV) in motor neuron disease (MND).

Methods: 23 subjects were recruited. QOL (SF-36, chronic respiratory disease questionnaire, sleep apnoea quality of life index (SAQLI), vital capacity, and maximum respiratory pressures were assessed two monthly and polysomnography four monthly. Subjects were offered a trial of NIV with any of: (1) orthopnoea, (2) unrefreshing sleep or daytime sleepiness, (3) Daytime $\mathrm{PaCO}_{2}>45 \mathrm{~mm} \mathrm{Hg}$, (4) $\mathrm{SaO}_{2}<90 \%$ for $\geqslant 5 \%$ of the night, (5) apnoea hypopnoea index $>10$.

Results: The improvement in the SAQLI symptoms domain (most responsive index) after 1 month NIV (effect size: mean difference/SD), and compliance are shown. Results for subjects in whom only one criterion for NIV was met are in brackets.

\begin{tabular}{|c|c|c|c|c|c|}
\hline Criteria fo & r NIV & $\mathrm{n}$ & $\begin{array}{l}\text { Continued } \\
\text { Rx }\end{array}$ & $\begin{array}{l}\text { Compliance } \\
\text { h/day }\end{array}$ & $\begin{array}{l}\text { QOL effect } \\
\text { size }\end{array}$ \\
\hline \multirow[t]{2}{*}{ Symptoms } & Orthor & a 11 & 9 & 7.3 & 2.98 \\
\hline & Sleep & 12 & $8(0)$ & $6.0(3.2)$ & $\begin{array}{l}1.34 \\
(0.00)\end{array}$ \\
\hline \multirow{2}{*}{\multicolumn{2}{|c|}{$\begin{array}{l}\mathrm{PaCO}_{2} \uparrow, \mathrm{SaO}_{2} \downarrow \\
\mathrm{AHI}>10\end{array}$}} & $6(0)$ & 5 & 9.7 & 2.75 \\
\hline & & 7 (4) & $3(1)$ & 4.2 (2.0) & $\begin{array}{l}0.33 \\
(-0.18)\end{array}$ \\
\hline
\end{tabular}

10/16 subjects starting NIV elected to continue. Among these, orthopnoea was the commonest indication (9/10). Moderate/severe bulbar weakness was associated with lower compliance (4.22 v 8.47 $\mathrm{h} /$ day) and less improvement in QOL (effect size: 1.85 v 3.28 ).

Conclusions-Orthopnoea was the best predictor of compliance with and benefit from NIV in MND. Moderate/severe bulbar involvement was associated with poorer (although still clinically useful) compliance and response.

\section{RESPONSIVENESS OF QUALITY OF LIFE INSTRUMENTS AND DOMAINS TO NON-INVASIVE VENTILATION IN MOTOR NEURON DISEASE}

SC Bourke, PJ Shaw, R Bullock, GJ Gibson University of Newcastle, Newcastle; University of Sheffield, Sheffield; Newcastle General Hospital, Newcastle, UK

Background: As a prelude to a randomised controlled trial of non-invasive ventilation (NIV) in motor neuron disease (MND), we studied which quality of life (QOL) instruments are most responsive. We report the results of an efficacy analysis of the impact of NIV on QOL in 10 subjects with MND.

Methods: QOL and functional status were assessed at 0,1 , and 3 months after initiation of NIV, then 2 monthly intervals using SF-36, general wellbeing schedule (GWbS), chronic respiratory disease questionnaire (CRDQ), sleep apnoea quality of life index (SAQLI), Epworth sleepiness scale (ESS), and ALS functional rating scale (ALSFRS). The maximum improvement in each index was assessed by effect size (mean difference/SD).

Results: Effect sizes for all responsive instruments are shown.

The GWbS and SF-36 energy vitality and health change showed non-significant improvements. The largest improvements were in domains assessing sleep related symptoms, which were maintained for a mean $\geqslant 320$ days (Kaplan-Meier). As expected the SF-36 physical function domain and ALSFRS declined with disease progression.

Conclusion: NIVimproves QOL in selected patients with MND. QOL domains assessing sleep related symptoms were most responsive, and the improvement was maintained at or above baseline for $\geqslant 320$ days despite disease progression. 


\begin{tabular}{|llll|}
\hline \multicolumn{1}{|l}{ Instrument } & Domains & Effect size & $\mathrm{p}$ Value \\
\cline { 2 - 4 } ESS & & 0.85 & 0.019 \\
SF 36 & Emotional limitation & 0.93 & 0.023 \\
& Mental health & 1.11 & 0.046 \\
CRDQ & All domains & $0.71-0.90$ & $0.018-0.044$ \\
SAQLI & Social isolation & 0.96 & 0.024 \\
& Symptoms & 1.77 & 0.0022 \\
& Score & 1.11 & 0.030 \\
\hline & & & \\
\hline
\end{tabular}

\section{THE EFFECT OF CANNABIS BASED MEDICINAL EXTRACT ON LOWER URINARY TRACT DYSFUNCTION IN ADVANCED MULTIPLE SCLEROSIS: PRELIMINARY RESULTS}

CM Brady, R DasGupta, OJ Wiseman, CM Dalton, KJ Berkley, CJ Fowler National Hospital for Neurology and Neurosurgery, Queen Square, London, UK

Aims: The primary aim of this open label pilot study is to evaluate the safety, tolerability, and efficacy of two preparations of sublingual cannabis based medicinal extract (CBME) in patients with advanced multiple sclerosis (MS) and refractory lower urinary tract symptoms (LUTS).

Methods: Patients with advanced MS (Kurtzke score $\geqslant 6.5$ ) and refractory LUTS (detrusor hyperreflexia demonstrated by cystometry) are eligible for recruitment into this ongoing study. Frequency-volume incontinence charts and pad testing are completed during the run in $(3$ weeks) and treatment (16 weeks) periods. For the first 8 weeks of treatment patients receive $\mathrm{CBME}$ containing equal amounts of cannabidiol (CBD) and tetrahydrocannibinol (THC) whereas a THC only preparation is prescribed for weeks 9-16. Patients self titrate the CBME until urinary symptoms are relieved or unacceptable side effects occur. To assess the acute effects of CBME, cystometry is performed and cannabinoid concentrations are taken before and after maximum tolerated dose of CBME at 4,8 , and 16 weeks on treatment.

Results: We report results of the first eight patients $(2 \mathrm{M}: 6 \mathrm{~F}$, age 31-64). A paired t test was used.

\begin{tabular}{|llll|}
\hline & & & \\
\hline & $\begin{array}{l}\text { Baseline } \\
\text { (3 weeks) }\end{array}$ & $\begin{array}{c}\text { CBD:THC:1:1 } \\
\text { (Weeks 1-8) }\end{array}$ & $\begin{array}{l}\text { THC } \\
\text { (Weeks 9-16) }\end{array}$ \\
\hline $\begin{array}{l}\text { Mean daytime frequency } \\
\begin{array}{l}\text { Mean episodes of } \\
\text { nocturia }\end{array}\end{array}$ & 10.0 & 8.0 & 6.9 \\
$\begin{array}{l}\text { Mean number of } \\
\text { incontinent } \\
\text { episodes/24h }\end{array}$ & 2.6 & 1.6 & 1.5 \\
\hline & 2.3 & 1.3 & 0.7 \\
\hline
\end{tabular}

The average cystometric capacity increased from $265 \mathrm{ml}$ at baseline to $439 \mathrm{ml}$ at 8 weeks, and to $353 \mathrm{ml}$ at 16 weeks after dosing with $C B M E$ in the department.

Conclusions: These preliminary results indicate that CBME may prove to be an effective additional treatment for refractory urinary symptoms in a selected group of patients with advanced MS.

\section{INTRONIC AND MISSENSE MUTATIONS WITHIN THE LAMP-2 GENE IN DANON DISEASE (X LINKED VACUOLAR CARDIOMYOPATHY AND MYOPATHY)}

NP Davies, C Beesley, PM Elliott, J Holton, B Lake, DN Landon, P Lee, H Mundy, B Winchester, MG Hanna Institute of Neurology, Queen Square, London; Institute of Child Health, London, UK

Danon disease is an $\mathrm{X}$ linked disorder characterised by cardiomyopathy, vacuolar myopathy, and variable mental retardation. It is now known to be due to mutations within a gene encoding the lysosomal associated membrane protein LAMP-2 on Xq24.

We report three families with Danon disease. Affected males in each family presented with profound cardiac symptoms but had mild proximal myopathy. Mental retardation was apparent in only three out of five affected males. Proximal myopathy and mental retardation were not apparent in affected females. Age at death was 15-24 years for men and $29-40$ years for women. Muscle biopsies revealed the characteristic vacuolar myopathy. We also showed intense deposition of the complement membrane attack complex (MAC) on vacuolated fibres. This immunoreactivity has been previously reported to be specific for another vacuolar myopathy ( $X$ linked myopathy with excess autophagy, XMEA). Genetic analysis revealed three new mutations in the LAMP-2 gene. We report the first missense mutation in LAMP-2 (W321R). In addition, two new intronic mutations were detected.

In summary, we confirm the clinical and pathological characteristics of Danon disease and suggest that deposition of MAC on vacuolated fibres may be a feature. We identified three previously unreported mutations in the LAMP-2 gene. The W321R mutation represents a new mutational mechanism, as all other mutations disrupt splicing or lead to premature truncation of the protein. In view of the sparse skeletal muscle signs in this disorder, we would recommend a skeletal muscle biopsy in any young patient with an apparently isolated cardiomyopathy.

\section{GLUTEN ATAXIA AND GLUTEN NEUROPATHY: THE EFFECT OF A GLUTEN-FREE DIET}

M Hadjivassiliou, RA Grünewald, AK Chattopadhyay, RH Kandler, JA Jarratt, GAB Davies-Jones The Royal Hallamshire Hospital, Sheffield, UK

We studied the effect of gluten free diet on patients with ataxia and/or peripheral neuropathy associated with gluten sensitivity.

Sixteen patients with gluten ataxia (sporadic ataxia with circulating antigliadin antibodies) were assessed before and after 12 months treatment with a gluten free diet. Subjective assessment of clinical state by visual analogue scale improved from mean score (SD) at baseline $0.49(0.17)$ to $0.7(0.26)$. When compared with a heterogenous control group of eight ataxic patients without gluten sensitivity or who declined treatment with a gluten free diet, the improvement was significant (repeated measures ANOVA $p=0.002$ ). Improvement was also seen in time standing with feet together without corrective movement (baseline 19.1 seconds (22.7), 12 months 31.2 seconds (26.4), $\mathrm{p}=0.05$ Student's $t$ test). There was a trend towards improvement in computerised measures of latency and accuracy of arm coordination.

Sural sensory action potentials were measured in a group of 13 patients with gluten neuropathy before and 12 months after starting a gluten free diet. Baseline and 12 month repeat measurements were also made in a control group of five subjects with gluten neuropathy who declined the diet. There was some increase in amplitudes in the group treated with diet: $1.4 \mu \mathrm{V}(1.3) \vee 2.3 \mu \mathrm{V}(2.1)$ but none in the control group : $1.6 \mu \vee \vee 1.1 \mu \vee(1.2)$. The change in amplitude between the two groups was not significant $(p=0.067$ MannWhitney).

These preliminary data suggest that a gluten free diet may be of benefit in some patients with neurological manifestations of gluten sensitivity.

\section{HUMAN COMPLICATION OF THE FOOT AND MOUTH CRISIS}

KAC Harkness, TG Staunton Norfolk and Norwich Hospital, Norwich, UK

We report an unusual complication of the foot and mouth crisis. After our patient, a marksman, had examined a culled bull for signs of life, the animal's head moved, trapping his feet and causing him to lose balance. He fell backwards onto the horn of another dead animal, sustaining a penetrating injury to the occiput. He did not lose consciousness but complained of loss of vision in the right hemifield, 30 minutes after the initial injury. In the neurosurgical unit CT demonstrated a left comminuted depressed occipital skull fracture with some impingement of the left occipital-parietal lobe. The patient was managed conservatively with intravenous antibiotics and discharged 1 week later.

Two weeks later the patient developed occipitofrontal headache with photophobia and presented to our neurology unit. He was afebrile, no meningism, no papilloedema, a resolving right field defect was noted. MRI and MRA images were performed. The vertebral arteries were normal. A left occipital skull fracture with an organising extradural haematoma indentating the left occipital lobe was demonstrated. Abnormal high signal was seen within the sagittal sinus. MRV demonstrated venous thrombosis occluding the posterior two thirds of the sagittal sinus. The patient was anticoagulated and symptoms resolved in a few days.

\section{A UNIQUE MODEL OF LISSENCEPHALY}

L Kinton, KA Barth, C Howart, JS Duncan, M Gardiner, NW Wood, S Wilson Institute of Neurology, Queen Square, London; University College, London, UK

Disorders of neuronal migration are important causes of drug resistant epilepsy. Mutations in two genes LIS1 and DCX have been shown to cause one such condition, agyria/pachygyria/band heterotopia complex. 
The zebrafish, Danio rerio, is used as a model organism for studying development of vertebrate systems including the CNS. Advantages of this model organism include rapid external development, transparency, and most recently morpholino antisense oligonucleotide technology for creating targeted gene knockdown. Conservation of genetic pathways across vertebrates make it a valid model organism.

The zebrafish orthologue of the LISI gene has been cloned and it shows a high degree of sequence conservation with human LIS $1(81 \%$ nucleotide, $93 \%$ amino acid identity). The expression pattern in the developing zebrafish is analagous to mammalian expression including high levels in the developing CNS.

The novel technique of morpholino antisense oligonucleotide injection has been performed against the lis 1 gene. Graded reduction in lis 1 protein translation and hence activity in the zebrafish embryo results (knockdown), providing an in vivo model of lissencephaly. These embryos show abnormalities in the developing brain, most notably the telencephalon and hindbrain. This is the first time this technique has been used to model a neurological disorder important in epilepsy pathogenesis.

\section{USE OF INTERNET MEDICAL WEBSITES AND OF NHS DIRECT BY NEUROLOGY OUTPATIENTS BEFORE CONSULTATION}

AJ Larner The Walton Centre for Neurology and Neurosurgery, Liverpool, UK

Objectives/methods: Two sources of medical information have recently become available to the general public: medical websites on the Internet, and the NHS Direct telephone helpline. This study measured the use of these resources before consultation by consecutive new referrals to general neurology outpatient clinics.

Setting: Two district general hospitals in north west England.

Results: Of 198 patients seen over 3 months, $66(33 \%)$ had home access to the Internet. Of these $66,15(22 \%=7.6 \%$ of all patients) had accessed websites containing medical information; only one patient volunteered this information.

Of these 15 , nine $(60 \%)$ accessed information which was adjudged inappropriate in the light of the final diagnosis. This was due to: incorrect self diagnosis, based on self directed searches (5/9); incorrect diagnosis by another doctor (2/9); other, pre-existing (correct) diagnoses not relevant to current symptomatology (2/9).

Internet users accessing appropriate information found that searches were time consuming and generally provided nothing beyond what they already knew. Only one patient found website information "very helpful", but this was inappropriate to the final diagnosis.

Only 4/198 patients (2\%) had telephoned NHS Direct before consultation; none volunteered this information. In only one did the call relate to neurological symptoms.

Conclusions: In this study around a quarter of patients with home access to the Internet used medical websites before neurological consultation; use of NHS Direct was negligible. Information accessed from Internet sites was adjudged inappropriate in $60 \%$ of cases. Internet searches, although seldom volunteered, may influence patients' health beliefs and expectations, and hence the subsequent consultation. Such considerations may become increasingly relevant as personal Internet access expands.

\section{AN IMPROVED METHOD OF QUANTIFYING ACTIVITY LEVELS IN NEUROLOGICAL PATIENTS}

C Lawthom, RMW van Deurson, CM Wiles University Hospital Wales, UK

Current assessments of mobility rely on brief observations of walking and questionnaire based scales that are surrogate measures of everyday activity. Accelerometry techniques now allow unobtrusive activity measurement over many days. Activity levels in two patients and two matched healthy controls were measured using the Stepwatch Activity Monitor, a device worn on the ankle to record cadence over 3 to 8 days, both at home and in hospital. An interviewer administered diary, the Rivermead mobility index (RMI), and a digital camcorder recording of gait (with calculation of stride length and hence distance) were also used

Patient 1 (female; 74 years) recovering from Guillain-Barré syndrome, was independent (RMI: $8 / 15$ ). The patient was active for on average 2.95 hours per day $(\mathrm{h} / \mathrm{d})$ ( range 1.3 to $4.1 \mathrm{~h} / \mathrm{d}$ ), and $18.43 \%$ of time awake: she walked an average of 1791 right steps covering a distance of $1593 \mathrm{~m}$ (range $857-2710 \mathrm{~m}$ ) Activity levels rose after discharge from hospital with a change in activity pattern from directed activity to less vigorous, frequent independent activity. Patient 2 (female; 34 years) with improving inflammatory cervical myelopathy, was also independent (RMI: 8/15). This patient had very limited activity which coincided with physiotherapy, with little

\begin{tabular}{|lllll|}
\hline Activity index & Patient 1 & Patient 2 & Control 1 & Control 2 \\
\hline $\begin{array}{l}\text { Mean activity } \\
\text { (h/day) }\end{array}$ & 2.95 & 0.87 & 5.80 & 4.90 \\
$\begin{array}{l}\text { Range of activity } \\
\text { (h/day) }\end{array}$ & $1.3-4.1$ & $0.8-1.4$ & $5.4-6.6$ & $3.6-7.1$ \\
\% time awake & 18.4 & 3.6 & 33.8 & 28.0 \\
$\begin{array}{l}\text { Right steps/d } \\
\text { (mean) }\end{array}$ & 1791 & 433 & 4245 & 3988 \\
$\begin{array}{l}\text { Mean distance/d } \\
\text { (m) }\end{array}$ & 1593 & 254 & 5282 & 5474 \\
Range of distance & $857-$ & $82-$ & $4152-$ & $2604-$ \\
(m) & 2710 & 501 & 6347 & 11172 \\
\hline & & & & \\
\hline
\end{tabular}

independent activity. As expected the two controls had far higher activity levels. Despite similar RMI scores, the patients showed very different activity levels: both showed reduced activity levels with walking, in one case virtually dependent on therapy. These data emphasise the wide range of normal activity levels in healthy and disabled people and the potential use of this technique in quantifying and monitoring mobility.

\section{SPECIFYING THE EXECUTIVE DEFICIT IN PARKINSON'S DISEASE; A COMBINED BEHAVIOURAL AND EVENT RELATED FMRI STUDY}

SJG Lewis, A Dove, R Cools, TW Robbins, RA Barker, A M Owen Cambridge Centre for Brain Repair, Cambridge, UK

Parkinson's disease (PD) is accompanied by a complex pattern of "frontal-like" impairments, which may include deficits in many aspects of working memory function. However, recent neuropsychological studies have questioned the psychological specificity of these "executive" deficits, while functional neuroimaging studies have been unable to identify the precise neural substrates responsible. In this study, two closely matched groups of patients with PD, differing only with respect to their performance on a standard test of executive dysfunction, were tested on a novel verbal working memory paradigm which allowed different aspects of performance to be assessed simultaneously, including maintenance, retrieval, and manipulation of the remembered information. The subgroup of patients with "executive" impairment were selectively impaired on the task, and specifically in those aspects of the test requiring the manipulation of information within working memory. These results suggest that it may be informative to functionally subdivide patients with PD according to whether their cognitive profile includes executive dysfunction or not; moreover, the specific nature of the impairment observed implicates the middorsolateral region of the frontal cortex as a likely neural substrate. To test this hypothesis directly, event related fMRI was employed in similar groups of PD patients performing the same verbal working memory task.

\section{GLUTEN ATAXIA IS IMMUNE MEDIATED}

D Mahad, K Oates, C Williams, R Grünewald, SJL Howell, MN Woodroofe, M Hajivassiliou Royal Hallamshire Hospital, Sheffield, UK

Gluten sensitivity is a state of heightened immunological responsiveness triggered by the ingestion of gluten in genetically susceptible individuals. Organ specific manifestations include an enteropathy (coeliac disease) a dermatopathy (dermatitis herpetiformis) and CNS involvement (gluten ataxia). Gluten ataxia accounts for up to a third of idiopathic sporadic ataxias. It is defined by the presence of antigliadin antibodies in otherwise unexplained sporadic idiopathic ataxia. The mechanism of neurological damage remains obscure.

Postmortem brain tissue of two patients with gluten ataxia showed T cell infiltration and upregulation of chemokine expression. CSF from 10 patients with gluten ataxia showed upregulation of the chemokine IP-10 when compaired with controls. Serum from patients with gluten ataxia stained human cerebellar Purkinje cells at dilutions up to 1:800 with strong staining seen up to 1:400. At 1:400 staining was not seen in the control groups (five patients with other causes of ataxia, five normal controls, five patients with coeliac disease). Commercial antigliadin antibodies stained human Purkinje cells in a similar manner. Persistent staining after adsorption with crude gliadin was only observed when using sera from patients with gluten ataxia.

These data provide further support to our contention that gluten ataxia is immune mediated. 


\section{EVIDENCE FOR DEMENTIA IN SPAST-HEREDITARY SPASTIC PARAPARESIS}

P McMonagle, P Byrne, S Webb, N Parfrey, M Hutchinson St Vincent's University Hospital, Dublin, Ireland

Objective: Cognitive impairment is recognised in SPAST-hereditary spastic paraparesis (HSP). It is unclear if this represents a dementia. Our aim was to examine SPAST-HSP pedigrees longitudinally for evidence of progressive cognitive decline.

Methods: Five families with SPAST-HSP were identified, 4 with detectable mutations and one based on linkage. Family members were tested for cognitive impairment at first visit and again after a minimum of 1 year. The Cambridge Cognitive Assessment (CAMCOG) has a maximum score of 107 , scores $<80 / 107$ indicate cognitive impairment. Non-parametric tests of significance were used.

Results: 34 patients over age 20 from five families had paraparesis. Repeat CAMCOGs were available for 22 . The age after 2.9 years follow up was 54.5 years. CAMCOG declined over that time from 86.6 to $82.4 / 107$ ( $p=0.018)$. In those with cognitive impairment at baseline $(n=9)$ CAMCOG declined further from 74 to $64.4 / 107$ $(p=0.012)$. The average age for this group was 66.4 years. CAMCOG for controls (age=65.9 years, $n=9$ ) showed little change (91.8 to $90.2 / 107, \mathrm{NS})$

Conclusions: We have demonstrated longitudinal cognitive decline in those with SPAST-HSP and further decline in those with established cognitive impairment, suggesting that this is a dementing process.

\section{TWO CLINICAL PRESENTATIONS OF ADULT ONSET RASMUSSEN'S SYNDROME SHARE COMMON IMMUNOLOGICAL AND PATHOLOGICAL FEATURES}

RS Nicholas, AC Scott, IK Hart The Walton Centre for Neurology and Neurosurgery, Liverpool, UK

Childhood Rasmussen's syndrome (RS) is a focal cortical inflammation causing epilepsy and progressive neurological deficits. We studied six patients to define the clinical and pathological features of adult RS and performed comprehensive screens for anti-CNS and other autoantibodies. All patients presented with epilepsy (average age 29 years). There were two patterns of clinical onset and progressionrapid (neurological deficit $\leqslant 12$ months after seizure onset; four patients) and slow (neurological deficit $\geqslant 42$ months after seizure onset). The rapid form presented with focal increased signal on T2 MRI that later developed into ipsilateral hemisphere atrophy. Histology was similar to childhood RS. In the slow form, MRI brain showed focal cortical atrophy. On histology, there was moderate perivascular $\mathrm{T}$ cell infiltration, microgliosis, and reactive astrocytosis. In both forms, there was bilateral focal cortical hypoperfusion on SPECT scans. All patients had multiple serum and CSF immune abnormalities and had similar HLA haplotypes. We propose that adult RS has two clinical phenotypes-rapid and slow-that share similar brain, CSF, and serum autoimmune abnormalities. Our findings may help the early diagnosis of adult RS and identify candidates for immunomodulatory therapy before they develop extensive, irreversible neuron loss.

\section{EPILEPSY SURGERY IN PATIENTS WITH ADDITIONAL PSYCHOGENIC SEIZURES}

M Reuber, G Fernandez, M Kurthen, J Schramm, CE Elger University of Bonn, Bonn, Germany

Objective: To assess whether surgery to reduce or control epileptic seizures is safe and effective in patients known to have additional psychogenic seizures.

Methods: We reviewed our computerised database of 1342 patients evaluated for epilepsy surgery and identified 13 patients with both epileptic and psychogenic seizures on whom postoperative data were available. Outcome data were gathered from patient records and completed by telephone interviews. Mean postoperative follow up was 56 months.

Results: Epilepsy surgery led to relevant improvements in 11 of 13 patients. Seven patients became completely seizure free, two patients became free of epileptic seizures but continued to have infrequent psychogenic seizures, one patient reported an over $80 \%$ improvement of epileptic seizure frequency and an abolishment of psychogenic attacks, in one patient non-disabling epileptic seizures persisted at lower frequency but psychogenic seizures stopped. In two of 11 patients, epilepsy surgery failed to produce notable improvements. Although epileptic seizures improved, their psychogenic seizure disorder remained highly disabling. In both of these patients pathological illness behaviour had been noted preoperatively.
Conclusion: A diagnosis of additional psychogenic seizures should not be considered an absolute contraindication to epilepsy surgery although patients should undergo careful preoperative psychiatric evaluation.

\section{EXPLORING THE CONNECTIVITY OF BAND HETEROTOPIA USING DIFFUSION TENSOR IMAGING}

FJ Rugg-Gunn, M Guye, SH Eriksson, G Parker, GJ Barker, C Wheeler-Kingshott, JS Duncan University College London, London, UK

Introduction: Diffusion tensor imaging (DTI) is an MRI method that evaluates the diffusion of water molecules. DTI tractography allows mapping of white matter tracts. Band heterotopia (BHT) is a malformation of cortical development in which there are bands of ectopic grey matter in the subcortical white matter. Previous functional imaging has shown activations in BHT. We used tractography in five patients with $\mathrm{BHT}$ to determine whether connecting tracts could be identified in vivo.

Methods: Five patients with epilepsy and bilateral BHT were scanned with DTI. Anisotropy (diffusion directionality) maps were calculated and evaluated using fast marching tractography. This produced maps of the likelihood of connection which were used to determine the pathways of white matter tracts through the areas of $\mathrm{BHT}$, and compared with control data.

Results: White matter tracts were identified passing from and through the BHT to the cortex in each patient.

Conclusions: Using tractography we have shown that white matter tracts traverse and connect BHT to overlying cortex. This shows the structural basis of the functional connectivity implied by activation studies and the absence of focal neurological deficit. This result demonstrates the potential of the method to indicate structural connectivity in vivo.

\section{THE PROGNOSTIC VALUE OF INTRATHECAL IMMUNE RESPONSE AGAINST AXONAL COMPONENTS IN PATIENTS WITH MULTIPLE SCLEROSIS}

OA Seidi, YK Semera, NA Gregson, MK Sharief Guy's Hospital, London, UK

Background: We have recently reported a significant correlation between clinical disability and intrathecal release of antibodies against axonal cytoskeleton in patients with primary and secondaryprogressive MS.

Objectives: To determine the prognostic value of intrathecal antibodies to axonal proteins for future development of disability in MS patients.

Methods: In a prospective study, we measured intrathecal levels of antibodies against actin, tubulin and neurofilament light-subunit from 51 patients with relapsing-remitting, secondary, or primaryprogressive MS. Patients were clinically monitored for 2 years after the lumbar puncture (LP). We included 52 patients with other neurological disorders and eight healthy subjects, as controls. We also measured antibodies against myelin basic protein (MBP) as a control antigen.

Results: Intrathecal levels of antibodies against all axonal proteins, but not anti-MBP, correlated with the expanded disability status scale (EDSS) score at the time of LP. Moreover, MS patients with high intrathecal levels of antibodies against axonal proteins accumulated more neurological disability at the end of the 2 year period compared with patients with low levels. By contrast, antibodies against MBP did not correlate with EDSS score at the end of the study.

Conclusion: The determination of intrathecal antibodies against axonal proteins may be clinically useful in MS patients.

\section{MAPPING THE DISEASE LOCUS IN A LARGE KINDRED WITH GENERALISED EPILEPSY}

A Siddiqui, M Davis, PH Dixon, M Johnson, M Koepp, SD Shorvon, JWA Sander, RM Gardiner, JS Duncan, NW Wood Institute of Neurology, Queen Square, London; University College London, UK

A large British family with epilepsy underwent careful clinical evaluation using a structured seizure questionnaire. There was similarity to the GEFS plus (generalised epilepsy and febrile seizures plus) phenotype in that affected individuals manifested a variety of childhood onset epilepsy phenotypes with or without preceding febrile seizures. A simulation analysis estimated a maximum lod score of 4.17 at theta $=0$ and 3.84 at theta=0.05. The known loci for GEFS plus on chromosome $19 q$ and $2 q$ and the febrile seizures loci on chromosome $8 q, 19 p, 2 q$, and $5 q$ were investigated initially and excluded with lod scores ranging from -2.01 to -infinity.

Genome wide linkage analysis was undertaken using a medium density linkage mapping set. This contained 400 fluorescent dye labelled microsatellite markers that defined a $10 \mathrm{cM}$ resolution index map. 
This work identified two main regions of linkage on chromosome 2 and 5 . These areas were investigated by genotyping further markers and constructing haplotypes. Two point lod scores are 3.01 at theta $=0$ and 2.41 at theta $=0$ respectively, and multipoint lod scores of 3.01 and 2.85 respectively. Searches are being carried out for candidate genes using the Draft Human Genome Browser.

\section{MAGNETISATION TRANSFER RATION AND N-ACETYL ASPARATE AS INDEPENDENT MARKERS OF MYELIN AND AXONAL INTEGRITY IN DEMYELINATING LESIONS: A MAGNETIC RESONANCE IMAGING STUDY OF CENTRAL PONTINE MYELINOLYSIS}

NC Silver, DG MacManus, CA Davie, DH Miller Institute of Neurology, University College London, UK

Magnetisation transfer ratio (MTR) measurement seems promising as a putative marker of demyelination in the CNS. Small MTR reductions may also occur as a result of axonal loss, oedema, or inflammation. The individual contribution of tissue components to MTR values in healthy tissue is unknown. Central pontine myelinolysis (CPM), a condition characterised by severe demyelination and occasional minimal axonal loss in the absence of inflammation or oedema, provides a useful in vivo model to assess the effect of myelin disruption on MTR.

Three patients with CPM were studied with magnetisation transfer imaging and proton magnetic resonance spectroscopy (MRS) on one or more occasions during their illness. Moderate to severe MTR reductions were observed in acute lesions and resolution of MTR parallelled clinical recovery. Marked MTR reductions were observed despite general preservation of $\mathrm{N}$-acetyl aspartate (NAA; an in vivo MRS marker of axonal integrity).

These observations support the hypotheses that (a) myelin forms the predominant contribution to MTR values in healthy white matter, (b) demyelination itself can result in severe MTR reduction, (c) profound MTR reduction may be seen in the absence of significant neuronal damage, (d) MTR and NAA provide relatively independent markers of myelin and axonal pathology, and (e) MTR and NAA may provide useful diagnostic and prognostic information in CPM.

\section{A PROSPECTIVE STUDY OF EPILEPSY SURGERY IN THE UK}

JK Solomon, A McEvoy, SD Lhatoo, ND Kitchen, SD Shorvon Institute of Neurology, Queen Square, London, UK

The treatment of epilepsy has been revolutionised by improvements in epilepsy neurosurgery. We wished to determine the number of consultant neurosurgeons performing epilepsy surgery in the United Kingdom and to evaluate prospectively the total number of epilepsy procedures performed.

All practising consultant adult and paediatric neurosurgeons in the United Kingdom were sent a postal questionnaire asking if they had performed epilepsy surgery in the last year. Each identified surgeon was then asked to record the type and number of surgical procedures they had performed in a questionnaire mailed to them at the end of every month for 6 consecutive months commencing February 2000.

Thirty two consultant neurosurgeons in current practice who performed epilepsy surgery were identified. Out of a total of 192 monthly questionnaires there was a response rate of $95 \%$. A total of 289 surgical procedures for epilepsy were performed, suggesting an annual figure of 578 procedures.

Although there has been an almost twofold increase in curative epilepsy surgery in the last decade, this still falls far short of what is required from epidemiological estimates. The need for more surgical assessment units as well as trained epilepsy surgeons is emphasised by our study's figures.

\section{DERMATITIS HERPETIFORMIS, COELIAC DISEASE, AND NEUROLOGICAL DYSFUNCTION}

DSNA Pengiran Tengah, DJ Unsworth, L Fry, G Giovannoni, A Church, B Turner, GKT Holmes, AJ Wills Queens Medical Centre, Nottingham; Southmead Hospital, Bristol; Imperial College London; Institute of Neurology, London; Derbyshire Royal Infirmary, Derby, UK

Objective: To investigate the prevalence of neurological abnormalities in patients with dermatitis herpetiformis (DH) and coeliac disease (CD) based on previous data suggesting that gluten is neurotoxic via immune mechanisms.

Methods: 35 patients with biopsy proved DH and 38 patients with biopsy proved $C D$ underwent thorough neurological examination and detailed case note review. Nerve conduction studies were requested where appropriate. The DH cohort was assessed for anti neuronal antibodies (Anti-Hu and Yo).
Results: Unexplained neurological abnormalities occurred in four patients with $\mathrm{DH}$ : chorea (one), essential tremor (one), and migraine (two); and nine patients with CD: epilepsy (one), migraine (six), benign fasciculations (one), and possible sensory neuropathy (one). Antineuronal antibodies were negative in all but one patient who had equivocally positive anti-Hu antibodies. An unexpected finding was the presence of a novel antispinal antibody in over $50 \%$ of these patients. The lifetime prevalence of essential tremor and migraine in these two cohorts was unremarkable. The patient with chorea had been on long term phenytoin, which is a reported association.

Conclusion: No cases of gluten ataxia were detected. The neurological abnormalities found in these patients are likely to be either chance or spurious associations contrasting with previous research. However, the unexpected discovery of this novel spinal antibody in DH patients requires further investigation, particularly in the light of previous studies suggesting an increased prevalence of sensory ataxia in patients with established gluten sensitivity.

\section{WHITE MATTER T1 RELAXATION TIMES IN MULTIPLE SCLEROSIS ACQUIRED USING THREE DIMENSIONAL MAGNETIC RESONANCE IMAGING}

L Vaithianathar, CR Tench, PS Morgan, LD Blumhardt Queen's Medical Centre, Nottingham, UK

T1 relaxation time (T1) mapping provides a quantitative method for assessing disease burden in multiple sclerosis (MS).

Previous studies have been limited to sampling small regions of normal appearing white matter or discrete lesions.

We compare total white matter Tl values in controls and MS patients from different clinical subgroups using histogram analysis. Relations between T1, disability, and T2 lesion volume are explored.

29 patients with MS $(11$ relapsing-remitting, RR; 11 secondary progressive, SP; seven primary progressive) and 11 healthy controls were recruited. High resolution T1 maps were acquired using a three dimensional fast low angle shot sequence. Dual echo T2 weighted images were also obtained. Disability was assessed using the expanded disability status scale (EDSS). Normalised white matter histograms were generated after extracting skull, cerebrospinal fluid and grey matter. T2 lesion volume was determined using a semiautomated technique.

Histogram standard deviation (SD) was significantly greater in each patient subgroup than in controls $(p=0.0001)$, and in patients with SPMS compared with RRMS ( $p=0.02)$. In the overall patient cohort, histogram SD correlated significantly with T2 lesion volume ( $r=0.79$, $p<0.0001)$, and disability $(r=0.53, p=0.004)$.

White matter T1 histograms evaluate global tissue damage in MS, and may provide a sensitive, objective measure for monitoring disease progression.

\section{OLIGODENDROCYTES SECRETE FACTORS WHICH DECREASE NEURONAL APOPTOSIS IN VITRO VIA $\mathrm{PI}_{3}$-KINASE/AKT SIGNALLING PATHWAYS}

A Wilkins, S Chandran, DAS Compston Cambridge Centre for Brain Repair, Cambridge, UK

There is increasing data both from experimental models and from pathological and clinical studies of multiple sclerosis showing that oligodendrocytes provide trophic support for neurons and withdrawal of that support may contribute to neuronal dysfunction. Understanding the nature of this support and underlying intracellular mechanisms may lead to development of therapeutic strategies to prevent neuronal/ axonal abnormalities occurring in multiple sclerosis. We have used an in vitro model to study neuronal-oligodendrocyte interactions.

Rat embryonic cortical neurons were cultured in media conditioned by oligodendrocytes at different stages of their lineage. Neuronal survival was measured by immunocytochemistry and by ${ }^{3} \mathrm{H}$ GABA uptake. Apoptosis was measured by TUNEL staining. Protein kinase analysis was studied by western blotting and immunocytochemistry.

Neurons showed a marked increase in survival when cultured in medium conditioned by oligodendrocyte precursor cells and differentiated oligodendrocytes, and a decrease in apoptosis, compared to non-conditioned media. This effect was blocked by inhibitors of $\mathrm{PI}_{3}$ kinase but not by MAP kinase inhibitors. Furthermore oligodendrocyte conditioned media caused an increase in Akt (protein kinase B) phosphorylation in neurons consistent with an anti-apoptotic effect.

This study provides evidence that soluble factors from oligodendrocytes support neuronal survival and decrease apoptosis via $\mathrm{PI}_{3}$ kinase/Akt signalling pathways. 